\title{
Validation of the multi-objective structural optimisation of a composite wind turbine blade
}

E. M. Fagan a,c,d, O. De La Torre ${ }^{\text {a,c,d, }}$, S. B. Leen ${ }^{\text {b,c,d }}$, J. Goggins ${ }^{\text {a,c,d }}$

${ }^{a}$ Civil Engineering, National University of Ireland Galway, Ireland.

${ }^{\mathrm{b}}$ Mechanical Engineering, National University of Ireland Galway, Ireland.

${ }^{\mathrm{c}}$ Centre for Marine and Renewable Energy (MaREI), Galway, Ireland.

${ }^{\mathrm{d}}$ Ryan Institute for Environmental, Marine and Energy Research, Galway, Ireland.

\section{Abstract}

Structural optimisation of a wind turbine blade is presented in this work. The optimisation was performed using a multi-objective genetic algorithm and finite element modelling to determine the optimal structural design for a glass fibre-reinforced polypropylene composite blade. A candidate blade design from the Pareto efficient set was manufactured and tested for a range of structural characteristics, including: mass, centre of gravity, deflections, strains and natural frequencies. Static testing was carried out using a Whiffle tree test rig and a laser scanner was used to determine the deflection of the blade to a high degree of accuracy. The finite element model results for the custom-made design are compared to the measured blade response. The FE model predictions for strains, mass and natural frequencies are in relatively good agreement with the test results; however, notable deviations in the deflections predictions are attributed to modifications to the blade for manufacture and the shell-based modelling approach. The differences are discussed in detail and recommendations for future design work are outlined. The test results of the bespoke blade are also compared to two additional designs to determine the level of improvement afforded by the genetic algorithm approach. The bespoke glass fibre blade demonstrated an improvement in tip deflection of $16 \%$ relative to the original blade design, with a slight decrease in mass. 
Keywords: optimisation, genetic algorithm, composite materials, wind turbine blade, structural testing; blade design

\section{Introduction}

Structural blade design can be considered as a combinatorial optimisation problem: a finite number of feasible solutions exist and could be solved by basic enumeration, however, the total number of these solutions is so great that an alternative method of finding the optimum is needed [1]. The required algorithm needs to find a solution that is as good as possible in a reasonable timeframe. Metaheuristics offers a search approach that meets these constraints. Metaheuristics defines a set of algorithms that combine (i) constructive algorithms that build upon past search experience and (ii) local search algorithms that explore the design space [2]. Metaheuristics covers a number of optimisation strategies that have been used extensively in the literature, including: Ant Colony Optimisation, Simulated Annealing and Evolutionary Computation. The group of algorithms contained within the term Evolutionary Computation were inspired by the processes of Darwinian evolution. Genetic Algorithms (proposed by Holland [3]) make up a significant proportion of these algorithms, which are typically applied to discrete combinatorial optimisation problems.

Genetic algorithms (GAs) apply functions that mimic genetic operations to sets of design variables. Hence, terms from microbiology permeate the language of GAs, for example: genes represent individual design variables, a chromosome refers to the set of design variables forming a potential solution, a population refers to the group of potential solutions and a generation represents each iteration of the algorithm. For a 
comprehensive discussion of the many formulations of GAs, from an engineering perspective, the review article by Marker and Arora [4] is recommended.

Chehouri et al. [5] reviewed optimisation strategies, objective functions and a wide range of design constraints for wind turbine blade design and found that the main optimisation methods were either gradient based approach (GBA) methods or metaheuristics. GBA methods (such as Kathiravan et al. [6] for example) can prove very effective at finding the optimum solutions to problems with large numbers of design variables; however, the large number of computations they require mean they are often more time consuming and they may prematurely converge to local optima [5], [7]. Of the metaheuristics, genetic algorithms and particle swarm optimisation (PSO) algorithms (for example [8]) were the most common. Design studies on composite plates have shown the effectiveness of optimising the ply angle and laminate thicknesses by applying failure criteria to determine the optimum structural performance within design constraints, e.g. Walker and Smith [9], Narayana et al. [10] and Almeida and Awruch [11]. Studies like these have demonstrated the benefits resulting from FE analyses paired with GAs and have led to optimisation studies of full wind turbine blade structures. By defining the order and distribution of the laminates that make up the blade structure as separate variables the entire structural design of the blade can be encoded as a permutation and/or combination problem to be tackled with a metaheuristic approach. Hence, optimisation analyses of blades typically focus on structural variables, including laminate thicknesses [8], ply orientations [12] and internal structural configurations [7]. Dal Monte et al. [13] used a multi-objective genetic algorithm to modify the distribution of the composite material in a $7.5 \mathrm{~m}$ long wind turbine blade. The result of the optimisation was a blade design with approximately $8 \%$ decrease in mass, $12 \%$ decrease in flapwise deflection and an 
undesirable $3 \%$ increase in edgewise deflection. The study highlighted the importance of the choice of objective function to ensuring that new blade designs perform as intended. Hu et al. [14] optimised the structural design of a blade for a 2 MW horizontal axis wind turbine. The design objectives minimised the mass and cost of the blade, while constraining the deflection and stress for an extreme wind gust loading. The 20year fatigue life (calculated using Miner's rule) was also incorporated as a constraint on the designs. In addition to structural optimisation, studies of wind turbine blades often investigate the aerofoil and blade geometric variables to improve the power production capacity and aerodynamic performance of the turbine [15] to [17]. The scope of the optimisation problem can be expanded to investigate the coupled aero-elastic behaviour of novel blade designs (e.g. Pourrajabian et al. [18]).

The present study is focused on the design of a wind turbine blade for a specific $15 \mathrm{~kW}$ turbine; therefore, the optimisation is constrained to the structural variables, such as the distribution of laminate thicknesses and ply drop locations in the blade, while retaining the original blade geometry. The optimisation technique used is based on the nondominated sorting genetic algorithm (NSGA-II) [19] and the GA objectives for each blade design are determined using the finite element software package Abaqus [20]. A previous design study [21] was performed on the composite blades, which identified several concept designs for a glass fibre-reinforced blade. In the previous study the multi-objective GA was used to optimise the structural design of the blade using the mass and tip deflection as the two objectives. The distribution of the thickness and orientation of the laminates throughout the blade was controlled by the design variables defined in the GA. The optimisation study in the present paper includes an extra design variable to control the width of the spar caps on the blade, thereby increasing the maximum stiffness attainable in the previous study. In the present work, a blade design 
is chosen from the set of designs generated by the GA and manufactured, with some modifications to adhere to the manufacturing procedure. The FE model predictions for blade characteristics such as blade mass, deflections and natural frequencies are compared to measured values and their differences assessed, thereby assessing the validity and assumptions of the optimisation methodology.

The previous study also reported test results for two blade designs, a glass fibrereinforced polypropylene blade (GFPP blade) and a hybrid blade made from GFPP with carbon fibre-reinforced spar caps (CFPP blade). The GFPP blade represents the original design for the wind turbine blade. Operational issues arose, however, due to the excessive flexibility of the design. The carbon-fibre reinforcement markedly improved the blade stiffness, although with the disadvantage of a considerable increase in manufacturing costs. Not alone was the carbon fibre material more expensive, the spar caps were separately cured prior to being laid up in the blade, complicating the manufacturing procedure. Therefore, the goal of the optimisation study is to improve the stiffness properties of the fully glass fibre-reinforced blade design and remove the requirement for using carbon fibre materials if possible. The test results from the two blade designs are compared to the results of the bespoke blade designed in the present study.

The goals of the study are:

(i) Use the multi-objective GA to identify a set of potential blade designs.

(ii) Manufacture and test a bespoke blade design from the optimum set.

(iii) Compare the FE predictions for the bespoke blade to the test results.

(iv) Compare the bespoke blade design to the original GFPP and hybrid GFPP-CFPP blades. 


\section{Methodology}

Section 2.1 outlines the experimental test set-up and instrumentation. Section 2.2 provides the details of the finite element modelling approach. Section 2.3 describes the multi-objective structural optimisation of the wind turbine blade. The method of encoding the structural design into a number of design variables is discussed and the objective functions used to evaluate the fitness of each potential blade design are provided. Finally, Section 2.3 describes the procedure of manufacturing the composite blade and the modifications to the design required to facilitate construction.

\subsection{Experimental Testing}

A Whiffle tree test rig was used in the experiments to apply a mechanical load distribution equivalent to the aerodynamic loading experienced by a turbine blade in operation. Figure 1 shows the Whiffle tree rig and the instrumentation used during testing. The load was applied to the blade at eight points, with the highest percentage of the load applied towards the tip of the blade. The load was applied by raising the Whiffle tree with the laboratory crane and was monitored using a Sensotec 50,000 lbs (222.4 kN) load cell (model 41/0573-01, linearity $\pm 0.1 \%$ full scale) connected between the rig and crane. The blade was instrumented with nine $120 \pm 0.5 \Omega$ (model Tokyo Sokki Kenkyujo Co. Ltd. FRA-6-11-3LT) electrical resistance rosette strain gauges along its length. The blade deflection was measured with two draw-wire displacement sensors (model Micro-Epsilon WDS-500-P60-CR-P) with a measuring range of 500 $\mathrm{mm}$ (linearity $\pm 0.1 \%$ FSO) and two draw-wire displacement sensors (model MicroEpsilon WDS-1000-P60-CR-P) with a measuring range of $1000 \mathrm{~mm}$ (linearity $\pm 0.1 \%$ FSO). The locations of the strain gauges and draw-wire displacement sensors (also known as stringpots) are provided in Table 1. Three linear variable displacement 
transducers (LVDTs) with a $\pm 12.5 \mathrm{~mm}$ range (ACT500A with linearity $\pm 0.5 \%$ FSO) were used to monitor any displacement of the base plate during testing.

A Leica C10 ScanStation laser scanner was used to scan the blades before and after applying the static load. The scanner has a resolution of one point every $10 \mathrm{~cm}$ at the medium level of operation and one point every $2 \mathrm{~cm}$ at the highest level of operation, at a range of $100 \mathrm{~m}$. At an average distance of $3 \mathrm{~m}$ (typical of the present tests), the medium resolution scans resulted in a point measured every $3 \mathrm{~mm}$ and the highest resolution scans a point every $0.6 \mathrm{~mm}$. Modal tests were conducted to determine the flapwise natural frequencies of the blade using a Dytran model 5805A impulse sledge hammer with a one pound head (sensitivity $1 \mathrm{mV} / \mathrm{lbf}$ ) and three accelerometers (model ENDEVCO 752A12). Table 1 also shows the locations of the accelerometers on the blade. The total crane load is provided with the results in Section 3.

\subsection{FE modelling}

The FE models were generated using an in-house Python-based code called BladeComp. Inputs to the code include: the material properties (Table 2), the twist angle and chord distributions (Table 3), the layups and spar cap width (Table 4) and the loading on the blade. From these inputs, BladeComp automatically generates the FE models and conducts the non-linear geometric analyses, returning the values of the userdefined objective functions, for each blade design, to the multi-objective GA.

The blades were constructed from two types of glass fibre-reinforced polypropylene: biaxial $\left( \pm 45^{\circ}\right)$ woven Twintex plies and unidirectional Plytron plies. The elastic material properties of both glass fibre materials are provided in Table 2. Table 2 also contains the material properties for the carbon fibre-reinforced epoxy, which was used in the spar caps of the CFPP blade design. The blades were constructed with a single shear web in an I-beam configuration (see Figure 2). The blade geometry is defined by 
the distribution of the chord length and twist angle along the blade (Table 3). The root of the blade is slanted at approximately $50^{\circ}$ to the blade length to facilitate connection to the turbine hub; hence, all measurements are defined from the start of the slanted region at the leading edge of the blade (Figure 2). NACA 4415 airfoils were used along the entire length of the blade, transitioning to a rectangular section at the blade root. This results in a relatively thin blade with limited sectional stiffness.

The Whiffle tree rig applied load in the flapwise direction (perpendicular to the rotorplane) only and the loading applied to the FE models matched this distribution. The models were partitioned at eight locations along the blade, coincident with the application points in the test. The partitioned sections were then coupled to reference nodes using a structural distributed coupling constraint and the appropriate percentage of the total load was applied at each location. The root of the blade was fully constrained with an encastre boundary condition. The FE models used S4R 4-noded, reduced integration, linear shell elements and a mesh convergence study was performed as part of this work. The study resulted in a sufficiently refined mesh of approximately 25,000 elements. The average element measured approximately $10 \mathrm{~mm}$ per side in the spar caps and shear web, with slightly larger elements in the leading and trailing edge sections.

The effect of using reduced integration elements was also checked before the analysis was conducted. The impact of their use was negligible on the deflection predictions (values within $0.2 \%$ ) and similarly for the far field strain results (values within approximately 1\%). The S4 elements did predict higher values of strain near the root, where the web connects to the spar caps; however, future solid modelling of the blade in this region is required to capture the interaction of this geometry and the actual root-hub connection. 


\subsection{Multi-objective Optimisation}

The aims of the optimisation study were to maximise the stiffness of a glass fibre blade, while also minimising the blade mass. The optimisation study investigates the effects of varying the laminate thicknesses along the blade, as well as the locations of the ply drops and width of the spar caps.

\subsubsection{Design variables definition}

Figure 2 shows a cross-section of the blade, identifying five distinct regions: the spar caps (grey), the outer aerodynamic shell (white), the inner shell reinforcement on the trailing edge side (blue), the inner shell reinforcement on the leading edge side (blue) and the shear web (hatched red and white). The number of biaxial or unidirectional plies in each of these regions constitute the design variables for the optimisation study. The blade was broken down into five sections along its length and the locations of the ply drops were controlled by four design variables.

A total of 30 variables define the full blade structure in the analysis. Table 4 provides details on the design variables, including the description and the maximum and minimum values for each. Variables $x_{1}$ to $x_{5}$ correspond to the number of unidirectional plies in the spar caps of each blade section. Variables $x_{6}$ to $x_{10}$ correspond to the number of biaxial plies in the outer shell of each blade section. Variables $x_{11}$ to $x_{15}$ correspond to the number of biaxial plies in the internal trailing edge shell sections. Variables $x_{16}$ to $x_{20}$ correspond to the number of biaxial plies in the internal leading edge shell sections. Variables $x_{21}$ to $x_{25}$ correspond to the number of biaxial plies in the shear web sections. Variables $x_{26}$ to $x_{29}$ correspond to the distances from the root of the blade to each of the four ply drop locations and variable $x_{30}$ corresponds to the width of the spar caps (which remains constant along the blade). The design variables define the blade layups up to approximately $0.9 \mathrm{~L}$. 
Since the relatively thin aerodynamic profiles limit the thickness of the laminates in the blade, blade designs with an unrealistic overlap of material at the spar caps could potentially be generated. Hence, an initial check for overlap of the spar caps was performed for each blade design in the analysis. If the laminate thicknesses at a blade section exceeded the available internal space, a repair function modified the number of plies in that blade section. Further discussion of modifications to the blade designs required for manufacture is detailed in Section 2.4.

\subsubsection{Objective functions}

Two objective functions were defined for the present study, one for tip deflection and one for blade mass. The objective functions are defined as,

$$
\begin{array}{ll}
\text { Minimise } & f_{1}(\boldsymbol{x})=\delta_{t i p}(\boldsymbol{x}) \\
\text { Minimise } & f_{2}(\boldsymbol{x})=M(\boldsymbol{x})
\end{array}
$$

where $\delta_{\text {tip }}(\boldsymbol{x})$ is the tip deflection of the blade, $M(\boldsymbol{x})$ is the mass of the blade and $\boldsymbol{x}$ is the vector of design variables (Table 4).

\subsubsection{Genetic algorithm parameters}

The operating parameters of the GA were:

(i) A total of 20 blade designs in the population.

(ii) An analysis run time of 150 generations.

(iii) A variable chance of mutation from $3 \%$ to $5 \%$ for each design variable.

For further details of the operation of the algorithm (including crossover, mutation and repair strategies) refer to Fagan et al. [21].

\subsection{Bespoke Blade Construction and Modifications}

While the optimisation methodology resulted in blade designs with improved structural performance, many of the designs generated also added a significant level of complexity 
to the construction process, which would increase labour costs in a production blade. An issue that became apparent during the manufacturing process, was that the ply thickness used in the models was defined for the consolidated and cured composite. The bulk composite before curing was significantly thicker and, to fit the laid-up blade into the mold, some modification of the spar caps was necessary. The solution involved the removal of material from the trailing edge side of the spar cap plies to facilitate the layup. Figure 3 shows an example of the modifications to the UD spar cap plies. The UD plies were trimmed to remove up to $40 \mathrm{~mm}$ of material from the innermost 8 plies and $15 \mathrm{~mm}$ of material from the next 4 plies, while the outer 8 plies were not modified. Figure 4 shows these modifications to the actual plies. The figure also shows how the ply drops in the blade were staggered over 10 to $15 \mathrm{~mm}$ to reduce stress concentrations from large changes in thickness.

The custom-made blade design also resulted in relatively thin leading and trailing edge shell sections at the root of the blade. Therefore, four additional plies of biaxial Twintex, $300 \mathrm{~mm}$ wide, were added to the leading and trailing edge regions at the blade root (as shown in Figure 3 (a)). In addition to providing reinforcement in this region of the blade, the extra plies also equalise the thickness of the blade section and, hence, reduce stress concentrations around the bolt holes.

Once the modifications to the design had been confirmed, the blade was hand laid-up in the following nine steps:

(i) The outer layers of Twintex were laid into the mold.

(ii) The unidirectional Plytron plies were laid up.

(iii) The internal Twintex plies were laid up.

(iv) The Twintex shear web (which had been preconsolidated) was positioned.

(v) The internal bagging was placed around the shear web. 
(vi) The internal Twintex plies were folded over.

(vii) The opposite spar caps were laid up.

(viii) The outer Twintex plies were folded over and the mold closed.

(ix) The internal bag was pressurised and the blade cured in an autoclave.

Figure 5 shows several of the steps in the manufacturing process. The Twintex plies were cut to size so they overlapped slightly on the compression side of the blade.

\section{Results and Discussion}

Section 3.1 examines the results of the optimisation study and specifies the blade design for manufacture. The results of the study are also compared to the previous optimisation study, highlighting the effects of changing certain design variables. The primary goal of this study is to determine the effectiveness of the genetic algorithm based multiobjective design methodology. The validation of the methodology is discussed in Section 3.2 using a comparison of the FE model predictions and the experimental results for the GA-generated blade design, for a range of structural characteristics. In order to assess the accuracy of the models generated by the optimisation process, the FE model presented has not been modified with the changes to the spar cap laminates or root laminates described in Section 2.4. Some of the discrepancies between model and experiments can, therefore, be attributed to the changes required to produce a manufacturable blade. The goal of the comparison is to determine how accurately the genetic algorithm predicts final blade characteristics and whether an additional analysis step including more complex FE models is required in the process. In Section 3.3, a comparison of the bespoke blade design with the experimental results of the original GFPP and CFPP blades is performed. 


\subsection{Optimisation Results}

Figure 6 presents the results from the multi-objective optimisation performed in the previous study [21], highlighting the potential improvements in blade designs from the original glass fibre (GFPP) blade tested. The GA determined the Pareto efficient set of solutions for the design problem, i.e. the solutions forming the lower boundary in Figure 6. Extending lines of constant mass and constant stiffness from the test blade splits the solution space into quadrants based on structural performance. The green quadrant indicates blade designs lighter than the test blade and with improved stiffness. The yellow quadrants indicate designs that are either lighter or stiffer, but not both. Finally, the red quadrant indicates designs both heavier and less stiff than the test blade. The initial population in the GA was randomly generated, leading to the spread of designs in the top-right quadrant. As the algorithm progressed, the majority of the blade designs were found in the lower two quadrants.

The results of the optimisation study in the present and previous work are plotted in Figure 7. The tip deflection results are normalised by the length of the blade, $L$. The experimental test results from both the GFPP and CFPP blades are also included in the figure. The significant increase in blade stiffness due to the carbon fibre plies is evident. The CFPP blade also contained more glass fibre material than the original GFPP blade, resulting in the higher mass value. The optimisation results from the present study show significant potential improvement in blade stiffness compared to the previous work. The minimum tip deflection achievable in the previous study was approximately $0.16 \mathrm{~L}$, while the present study generated designs with tip deflection as low as $0.12 L$ (a $25 \%$ improvement). This improvement is attributed to the inclusion of the spar cap width as a design variable. 
Figure 7 also shows the "optimum" or bespoke blade design chosen for manufacture (orange circle). The design was chosen based on its position between the two experimental blades. The new blade design has a tip deflection of approximately 0.131 $\mathrm{L}$ and a mass of $20.4 \mathrm{~kg}$. This demonstrates a $30 \%$ decrease in tip deflection from the original experimental GFPP blade, with a $10 \%$ increase in mass. The design has a slightly higher tip deflection than is achievable in the full set of designs, however, it demonstrates a 5\% reduction in blade mass from the CFPP blade. The values of the GA blade design variables are included in Table 4. Predictably, the stiffer UD Plytron plies (variables $x_{1}$ to $x_{5}$ ) were maximised in the chosen design, along with the width of the spar caps $\left(x_{30}\right)$. The less stiff Twintex material $\left(x_{6}\right.$ to $\left.x_{10}\right)$ was minimised throughout the blade, though some reinforcement in the leading and trailing edge internal sections was retained ( $x_{11}$ to $\left.x_{20}\right)$. The shear web thicknesses were quite high along the length of the blade $\left(x_{21}\right.$ to $\left.x_{25}\right)$ and the ply drop locations ( $x_{26}$ to $\left.x_{29}\right)$ all occurred in the outer $50 \%$ of the blade length. The chosen blade design has a tip deflection of approximately $0.131 \mathrm{~L}$ and a mass of $20.4 \mathrm{~kg}$. This blade design demonstrates a $30 \%$ decrease in tip deflection from the original experimental GFPP blade, with a $10 \%$ increase in mass. Table 5 shows the mass and tip deflection values for the original GFPP and CFPP test blades from the previous study [21], and the bespoke FE blade design. This blade provided the initial basis for a design document issued to the blade manufacturers [23]. The thickness of the laminates along the centreline of the GFPP, CFPP and bespoke blades is shown in Figure 8. The increase in thickness at different distances along the blades is due to the additional internal plies in the leading and trailing edge sections. The most prominent difference between the two test blades and the GA generated design is the significantly lower thickness of the plies at the root of the new blade. 
A total of 3020 blade designs were generated by the algorithm (indicated by the yellow markers in Figure 7). The number of designs between $18.5 \mathrm{~kg}$ and $21.5 \mathrm{~kg}$ and with tip deflection less than $0.13 \mathrm{~L}$ was 250 (or approximately $8 \%$ of the total designs generated). These represent the set of blade designs for choosing the design of the bespoke blade. Since a repair function was used to modify the design variables in the cases where infeasible solutions were generated, a low number of infeasible blade designs were found, 105 in total (or $3.5 \%$ of the total designs). Of the 2915 feasible blade designs generated in the optimisation, the mean and standard deviation of the blade mass was $21.2 \mathrm{~kg}$ and $4.9 \mathrm{~kg}$ respectively and for the tip deflection was $0.15 \mathrm{~L}$ and $0.05 \mathrm{~L}$ respectively. Comparing the mean values for the population to the mass and tip deflection of the original GFPP blade design indicates that: (i) the average blade produced by the GA was about $15 \%$ heavier than the original blade and (ii) the average blade demonstrated about $20 \%$ lower tip deflection. Since the goal of the optimisation was to increase the stiffness of the glass fibre blade, the associated increase in blade mass was considered an acceptable trade-off.

\subsection{Comparison of FE Model and Experimental Results}

The finite element blade model was compared to the test blade in terms of values of: (i) mass and centre of gravity, (ii) deflection, (iii) longitudinal and transverse strain and (iv) flapwise natural frequencies. The results for the deflections and strains are shown for a total crane load of approximately $500 \mathrm{~N} \pm 1 \%$.

The predicted mass of the blade from the FE model was $20.4 \mathrm{~kg}$, compared to the actual blade mass of $18 \mathrm{~kg}$ (over predicted by 13\%). The mass of all of the plies in the blade was recorded during construction, allowing a direct comparison between the FE model and test blade for each section and material. The total mass of Plytron in the spar caps of the test blade was $7.45 \mathrm{~kg}$ and $8.31 \mathrm{~kg}$ in the model (over predicted by $11.5 \%$ ). 
However, the modifications to the spar cap thickness outlined in Section 2.3 were not accounted for in the FE model. Accounting for this reduction in thickness resulted in the FE model over predicting the mass of Plytron by approximately 5\%.

The mass of Twintex used in the blade was in close agreement: $8.82 \mathrm{~kg}$ in the test blade and $8.79 \mathrm{~kg}$ in the FE model (under predicted by $0.4 \%$ ). However, the Twintex rib showed significant disagreement, with the FE model over-predicting the mass by $80 \%$. This was attributed to use of shell elements in the FE model. The shell element approach resulted in a non-physical overlap of material at the connection between the spar caps and shear web. Adjusting for the overlap reduces the over prediction of the blade mass by the FE model to approximately $0.9 \mathrm{~kg}$ (5\%). Figure 9 shows the mass values of the experimental test blade and the unadjusted and adjusted FE model results. The centre of gravity of the test blade was measured at $0.35 \mathrm{~L}$, while the FE model location was $0.37 \mathrm{~L}$. This difference was as expected, since the FE model included the extra spar cap material which was predominantly removed from the outer half of the blade.

Several sources for the discrepancy in blade mass predictions were cited in the previous study, including the overlap between adjacent Twintex plies and the inclusion of nonstructural bagging material. In the present study, the mass of Twintex, Plytron and bagging material were all measured during manufacture and the blade was constructed with minimal ply overlaps; hence, these sources of error have been accounted for. The variation in fibre volume content and, hence, material density, may account for some of the remaining differences between model and test blades.

The longitudinal and transverse strain at 9 locations along the length of the blade were recorded using strain gauge rosettes. The FE model predictions at the same locations are compared to these in Figure 10. The predicted strain is clearly in good agreement with 
the experimental results along the entire blade, with the largest over predictions of $20 \%$ in longitudinal strain and $12 \%$ in transverse strain at the first strain gauge location $(0.12 L)$

The deflections were measured using four draw-wire displacement sensors. The experimental deflections and FE model predictions are plotted in Figure 11. Both deflections show the same trend; however, the FE model under-predicts the test results by approximately $18 \%$ at the tip. The LVDTs measured a small vertical deflection at the base plate, which resulted in a correction to the tip deflection results of up to $35 \mathrm{~mm}$. The lower than predicted stiffness of the test blade was attributed to the removal of UD material from the spar caps, thereby reducing the stiffness of the I-beam section in the outer half of the blade. Additionally, the excess stiffness of the FE model was attributed to the issues modelling the shear web. The extra material assumed to be present near the blade surface added to the stiffness of the section.

While the draw-wire sensors measured the absolute deflection of the four points on the blade, the laser scanner provided significantly higher precision measurements and was used to determine the 3D displacements. Figure 12 shows the processed point cloud results from the laser scanner for the blade loaded at $500 \mathrm{~N} \pm 1 \%$. The figure shows the deformed and undeformed blades overlaid upon one another. The draw-wire sensors were also included in the figure to illustrate the magnitude of the deflection transverse to the blade length. The deflection of the blade tip in three directions is highlighted in the figure. The three directions were defined as: the vertical direction $\left(\delta_{1}\right)$, the transverse direction $\left(\delta_{2}\right)$ and the longitudinal direction $\left(\delta_{3}\right)$ (Figure 12). Figure 12 also shows the undeformed and deformed FE models for comparison.

The deflection of the blade at several points along its length in the three directions is shown in Figure 13, comparing the FE model and the experimental results. The 
positioning of the laser scanner during the test resulted in the highest resolution scan at the trailing edge of the blade. Therefore, the measurements and FE results along the trailing edge are reported in Figure 13 (the deflection results in Figure 11 are for the centreline of the blade on the tension side). While the deflections are under-predicted for all three directions, the FE model captures the longitudinal and transverse deflections in the blade. The relatively low deflections in the FE model at $0.2 \mathrm{~L}$ and $0.3 L$ (in Figure 13 and Figure 11, respectively) indicate that the root region or the rootbaseplate connection is inaccurate. Modifications to the root stiffness would have a considerable effect on the predicted tip deflection; hence, future work will assess these issues.

The flapwise natural frequencies of the blade were determined using an impact hammer and accelerometers. The results of the modal tests are shown in Figure 14, compared to the predictions from the FE model. The FE prediction for the first natural frequency matches the test result, the prediction for the second natural frequency is $4 \%$ lower than the recorded frequency and the third natural frequency is approximately $15 \%$ higher than the recorded frequency.

Future optimisation work on this blade design could incorporate an additional objective function to find blade designs free of the manufacturing modifications discussed. This objective function could be focused on a measure of the thickness of the material at the trailing edge of the blade, such that the GA would be directed away from this region of the design space.

Lekou et al. [24] performed a comparative study on the impact of the choice in modelling strategies (such as linear or non-linear analysis, shell or solid element models and load application methods) on the predictive capability of wind turbine blade computational modelling. The results indicated that the choice of either shell or solid 
element models resulted in a coefficient of variance of about $5 \%$ between the models for the flapwise deflection and up to about $15 \%$ for the edgewise values. The authors also found that the choice of loading method had a significant impact on the results, effecting the predictions of the static strength, fatigue strength and buckling capacity. The combined effect of the use of shell elements and the modifications to the blade design during manufacture have resulted in the errors in prediction of the final blade properties by the GA-generated finite element model.

A source of error in the static test results resulted from experimental difficulties with accurately loading the blade. The load was applied by raising the Whiffle tree rig with the laboratory crane. However, the level of control of the crane was limited, resulting in an error of approximately $\pm 1 \%$ for the applied load. This error propagates through to the deflection and strain results of Figure 10, Figure 11, Figure 13, Figure 15 and Figure 16. This results in the FE model under predicting the tip deflection of the test blade somewhere in the range of $17 \%$ to $19 \%$, for example. In addition, the blades were loaded to each of the load levels only once during the tests due to the issues with load control. Future work will examine a more accurate and consistent means of controlling the load during the experiments.

\subsection{Comparison of Blade Designs}

The mass values for the three blade designs are shown in Figure 17. The bespoke GFPP blade was $16 \%$ and $3 \%$ lighter than the original CFPP and GFPP blades, respectively. The spar caps in the original GFPP blade were approximately $60 \mathrm{~mm}$ wide (compared to $145 \mathrm{~mm}$ wide in the new design), combined with the slight drop in overall mass, so that the new blade has a significant redistribution of material from Twintex to Plytron. Rather nonintuitively, the mass of the CFPP blade was significantly higher than the original GFPP blade, even with the use of carbon fibre material in the spar caps. As 
described in the introduction, the goal of the CFPP blade design was to significantly increase the blade stiffness, resulting in an over-application of material throughout the blade.

The deflection of the three blades for an applied load of $500 \mathrm{~N} \pm 1 \%$ is shown in Figure 15. The shape of the deflected blade is consistent for the two original blades, with relatively little deflection up to $0.3 \mathrm{~L}$, and the majority of the deflection occurring in the outer half of the blades. The custom-made blade displays a more consistent gradient of deflection along its length. This performance is attributed to the fact that the thickness of the blade (Figure 8) is significantly lower at the blade root than in the other two designs. The thickness of the blade spar caps is constant up to approximately $0.6 L$ and increases between $0.6 \mathrm{~L}$ and $0.75 \mathrm{~L}$. The added stiffness in this region results in the lower curvature and the lower resultant tip deflection. The new GFPP design gives a decrease in tip deflection of approximately $16 \%$ from the original GFPP blade.

The longitudinal strain along the three blades for an applied load of $500 \mathrm{~N} \pm 1 \%$ is plotted in Figure 16. The strain in the GA-generated GFPP blade and the CFPP blade follows the same trend with maximum values occurring between $0.3 \mathrm{~L}$ and $0.45 \mathrm{~L}$. The strain in the original GFPP blade is less consistent, with large peaks at approximately $0.3 L$ and $0.7 L$. The bespoke blade was instrumented with a larger number of strain gauges than the previous two blades to more accurately capture the distribution of strain along its length.

Figure 18 presents the first three flapwise bending mode natural frequencies for all three blade designs. The first two natural frequencies for the two glass fibre blades are very close in magnitude (less than $1 \%$ difference between the two blades), while the there is a difference of approximately $18 \%$ for the third. The carbon fibre-reinforced blade is 
considerably stiffer than the other two designs, resulting in the higher values of natural frequencies. The differences between the new GFPP blade and the CFPP blade for the three frequencies are $49 \%, 65 \%$ and $30 \%$, respectively.

\section{Conclusions}

This paper presents an effort at the validation of a genetic algorithm (GA) based composite blade design methodology. The methodology used a multi-objective genetic algorithm to identify a candidate blade design with improved stiffness and mass characteristics, which was subsequently manufactured and tested. In order to successfully manufacture the blade several modifications to the laminates from the GA predicted blade design were required. The blade structural characteristics such as mass, centre of gravity and results from static and modal testing were used to validate the FE predictions of the bespoke blade.

The comparison between the mass of the bespoke blade and the FE model revealed several sources of inaccuracy. The modifications to the blade design to facilitate its manufacture resulted in a large proportion of the mass difference in the UD material. The remaining differences were likely due to the use of shell elements in the FE model. The thin aerodynamic profiles (combined with the low overall blade mass) resulted in a relatively large over-prediction of the material in certain sections of the blade, most notably the shear web. This effect would be minimised in larger blades, where the thickness of the laminates constitute a smaller proportion of the cross-sectional area. The following summarises the comparison between the physical blade and FE model:

- Once corrected for the manufacturing modifications, the FE model mass prediction was quite close to the actual test blade (approximately $5 \%$ higher). 
- The FE model predictions for the longitudinal and transverse strain at the strain gauge locations were in good agreement with the test results along the entire length of the blade.

- The predicted deflection of the FE model followed the same trend as the test results; however, the model under-predicted the tip deflection by approximately $18 \%$. The excess stiffness of the FE model is at least partially attributed to the extra spar cap material that was removed during manufacture.

- High precision laser scans of the loaded and unloaded blade were used to further investigate the three dimensional deflections of the blade. While the model predictions were lower than the test results, the FE model captured the deflections of the blade in the longitudinal and transverse directions.

- The prediction of the first three bending mode natural frequencies was in close agreement with the test results.

The predictions of the FE model were in relatively good agreement with the tests of the bespoke blade for some of the structural characteristics, however, the deflection results were significantly under-predicted. The sources of difference between the model and tests have been identified and constraints to reflect these issues will be introduced in future work on structural optimisation. Shell models were used in the optimisation due to their lower computational cost; however, solid models would likely significantly increase the fidelity of the predictions. One method of leveraging the advantages of each method would be to first use the shell models in the GA optimisation, followed by the generation of a solid model of the chosen blade for detailed structural analysis before manufacture. Future work will investigate the potential of this proposed methodology. The bespoke blade was also compared to two other blade designs, one constructed solely from glass fibre polypropylene (GFPP) and another from GFPP with carbon fibre 
epoxy spar caps (CFPP). The results of the optimisation indicated that, while the stiffness of the CFPP design was unattainable for a solely glass fibre blade, there was an opportunity for improvement in structural performance. The results of the comparison are:

- The new blade design resulted in a slight decrease in mass from the original GFPP blade and a significant decrease in mass from the CFPP design.

- The removal of spar cap material for manufacture resulted in less improvement in tip deflection than was predicted by the FE model. For a load of $500 \mathrm{~N}$, the FE model of the new blade showed a $30 \%$ reduction in tip deflection from the original GFPP design, while the experimental blade showed only a $16 \%$ reduction in tip deflection.

- The measurements of the longitudinal strain showed a smoother distribution in the new blade than in the original GFPP blade.

The objective of the analysis was to obtain a blade design consisting solely of glass fibre materials with improved stiffness from the original design. The carbon fibre design used in the comparison demonstrated a major improvement in stiffness on the original blade, however, a target value for improvement in stiffness was never defined. This makes the comparison of results between the three blades somewhat difficult to interpret, as to what constitutes a demonstrable improvement. A quantitative target value of blade stiffness would clarify this interpretation and also provide a useful constraint for the genetic algorithm. Using this value as a constraint could be achieved with a penalty function applied to the deflection objective function. Future work on the design of the blades for the $15 \mathrm{~kW}$ turbine will provide a more quantified approach to defining the ultimate objectives of the optimisation. 


\section{Acknowledgements}

This material is in part based upon works supported by the Science Foundation Ireland Marine and Renewable Energy Ireland (MaREI) research centre under Grant No.

12/RC/2302. It was also funded by a fellowship from the College of Engineering and Informatics, NUI Galway, and was supported by an NUI Travelling Studentship, 2014. The last author would like to acknowledge the support of Science Foundation Ireland through the Career Development Award programme (Grant No. 13/CDA/2200). Additional thanks are given to the technical staff at NUI Galway.

\section{References}

[1] Gen M and Cheng R. Genetic Algorithms and Engineering Optimization. Wiley Series in Engineering Design and Automation 2000.

[2] Blum C and Roli A. Metaheuristics in combinatorial optimization: overview and conceptual comparison. ACM Computing Surverys 2003; 35: 268-308.

[3] Holland HJ. Adaptation in Natural and Artificial Systems. The University of Michigan Press. Ann Arbor, MI 1975.

[4] Marler RT and Arora JS. Survey of multi-objective optimization methods for engineering. Struct Multidiscip Optim 2004; 26(6):369-395.

[5] Chehouri A, Younes R, Ilinca A and Perron J. Review of performance optimization techniques applied to wind turbines. Appl Energy 2015; 142: 361-88.

[6] Kathiravan R and Ganguli R. Strength design of composite beam using gradient and particle swarm optimization. Compos Struct 2007; 81(4): 471-479.

[7] Barnes RH and Morozov EV. Structural optimisation of composite wind turbine blade structures with variations of internal geometry configuration. Compos Struct 2016;152:158-167. 
[8] Chen J, Wang Q, Shen WZ, Pang X, Li S, and Guo X. Structural optimization study of composite wind turbine blade. Mater Des 2013;46:247-255.

[9] Walker M and Smith RE. A technique for the multiobjective optimisation of laminated composite structures using genetic algorithms and finite element analysis. Compos Struct 2003;62(1):123-128.

[10] Narayana Naik G, Gopalakrishnan S, and Ganguli R. Design optimization of composites using genetic algorithms and failure mechanism based failure criterion. Compos Struct 2008;83(4):354-367.

[11] Almeida FS and Awruch AM. Design optimization of composite laminated structures using genetic algorithms and finite element analysis. Compos Struct 2009;88(3):443-454.

[12] Paluch B, Grédiac M, and Faye A. Combining a finite element programme and a genetic algorithm to optimize composite structures with variable thickness. Compos Struct 2008;83(3):284-294.

[13] Monte AD, Raciti Castelli M, and Benini E. Multi-objective structural optimization of a HAWT composite blade. Compos Struct 2013;106:362-373.

[14] Hu W, Park D and Choi D. Structural optimization procedure of a composite wind turbine blade for reducing both material cost and blade weight. Eng Optim 2013;45:1469-87.

[15] Shen X, Yang H, Chen J, Zhu X, and Du Z. Aerodynamic shape optimization of non-straight small wind turbine blades. Energy Convers Manag 2016;119:266-278.

[16] Fischer GR, Kipouros T, and Savill AM. Multi-objective optimisation of horizontal axis wind turbine structure and energy production using aerofoil and blade properties as design variables. Renew Energy 2014;62:506-515. 
[17] Vucina D, Marinic-Kragic I, and Milas Z. Numerical models for robust shape optimization of wind turbine blades. Renew Energy 2016;87:849-862.

[18] Pourrajabian A, Nazmi Afshar PA, Ahmadizadeh M, and Wood D. Aero-structural design and optimization of a small wind turbine blade. Renew Energy 2015;87:837848.

[19] Deb K, Pratap A, Agarwal S and Meyarivan T. A fast and elitist multiobjective genetic algorithm: NSGA-II. IEEE Transactions on Evolutionary Computation 2002;6:182-197.

[20] ABAQUS. Abaqus documentation. Dassault Systemes, Providence, RI, 2016.

[21] Fagan EM, Leen SB, de la Torre O, and Goggins J. Experimental investigation, numerical modelling and multi-objective optimisation of composite wind turbine blades. J Struct Integr Maint 2017;2(2):109-119.

[22] Long AC. Design and manufacture of textile composites. Limited, Cambridge, UK: Woodhead Publishing, 2005.

[23] Fagan EM and Goggins J. Blade Design for Kingspan 15 kW Wind Turbine. Research Report No. MS2.3.3-NUIG-R-002.0. National University of Ireland, Galway, Ireland. 2017.

[24] Lekou DJ, Bacharoudis KC, Farinas AB, Branner K, Berring P, and Croce A. A Critical Evaluation of Structural Analysis Tools Used for the Design of Large Composite Wind Turbine Rotor Blades Under Ultimate and Cycle Loading. Proc 20th Int Conf on Comp Mat, ICCM20 Secretariat 2015. 


\section{Figure Captions}

Figure 1 - The Whiffle tree rig applied the load to the blade at eight locations distributed along its length. The instrumentation used in the test included: strain gauges, draw-wire displacement sensors, LVDTs, a load cell and a laser scanner.

Figure 2 - The design variables control the thickness of the main regions of the blade $\left(x_{1}\right.$ to $\left.x_{25}\right)$, the ply drop locations $\left(x_{26}\right.$ to $\left.x_{29}\right)$ and the width of the spar caps $\left(x_{30}\right)$ in the optimisation procedure.

Figure 3 - The blade design was modified to improve manufacturability. (a) The root region of the blade was reinforced with additional biaxial Twintex plies and (b) the width of the spar caps was decreased on the trailing edge side.

Figure 4 - Photos of the modifications to the spar cap plies, including: staggered ply drops to avoid stress concentrations and reduced width of the spar caps on the trailing edge side of the blade.

Figure 5 - The initial steps in the blade layup procedure. The outer layers of Twintex were laid into the mold followed by the UD Plytron (a), the internal layers of Twintex were then laid up (b), followed by the preconsolidated shear web (c).

Figure 6 - Results from the previous multi-objective optimisation study [21]. The GFPP blade is noted by the green triangle. Lines of constant mass and constant stiffness split the solution space into quadrants that aid in identifying a new blade design.

Figure 7 - Results from the multi-objective optimisation of the present (yellow markers) and previous (black markers) studies. The test results of the GFPP and CFPP blades are denoted by the green triangle and blue diamond, respectively. The orange circle indicates the blade design chosen for manufacture, resulting from the present study and the blue circle represents the experimental results from the manufactured blade.

Figure 8 - Comparison of the thickness of the laminates along the centreline of the three blades from the FE models.

Figure 9 - The experimental results and FE prediction of the blade mass. The value of the FE model prediction adjusted to reflect the manufacturing modifications is also included.

Figure 10 - The experimental results and FE prediction of the longitudinal and transverse strain at 9 locations along the blade.

Figure 11 - The experimental results and FE prediction of the deflection along the length of the blade.

Figure 12 - Point cloud results from the laser scans during testing and FE model deflection results. The unloaded and loaded blades are overlaid and the deflection of the tip in three directions is highlighted for $(a)$ the elevation, $(b)$ the plan and $(c)$ the end elevation view of the blade. 
Figure 13 - Comparison between the laser scanner and FE model deflections for six points along the trailing edge of the blade. The indices 1, 2 and 3 refer to the coordinate systems shown in Figure 12.

Figure 14 - The experimental results and FE prediction of the first three flapwise bending mode natural frequencies.

Figure 15 - Blade deflection results from the experiments for the original CFPP, GFPP and new GFPP blades.

Figure 16 - The longitudinal strain results from the experiments for the original CFPP, GFPP and new GFPP blades.

Figure 17 - Blade mass results from the experiments for the original CFPP, GFPP and new GFPP blades.

Figure 18 - The first three bending mode natural frequencies from the experiments for the original CFPP, GFPP and new GFPP blades.

\section{Tables}

Table 1 - Locations of the strain gauges, stringpots and accelerometers along the length of the blade and in the chord-wise directions.

\begin{tabular}{ccc}
\hline Stain Gauges & Distance Along Blade $(\boldsymbol{l} / \boldsymbol{L})$ & Chord-wise $(\boldsymbol{c} / \boldsymbol{L})$ \\
\hline SG1 & 0.12 & 0.04 \\
SG2 & 0.22 & 0.03 \\
SG3 & 0.30 & 0.03 \\
SG4 & 0.37 & 0.03 \\
SG5 & 0.47 & 0.02 \\
SG6 & 0.55 & 0.02 \\
SG7 & 0.59 & 0.02 \\
SG8 & 0.70 & 0.02 \\
SG9 & 0.83 & 0.02 \\
Stringpots & & \\
\hline SP1 & 0.31 & 0.02 \\
SP2 & 0.44 & 0.02 \\
SP3 & 0.60 & 0.02 \\
SP4 & 1.00 & 0.01 \\
\hline Accelerometers & & 0.03 \\
A1 & 0.36 & 0.02 \\
A2 & 0.81 & 0.02 \\
A3 & 0.99 & \\
\hline
\end{tabular}


Table 2 - Unidirectional material properties of the glass and carbon fibre composites making up the blades [22].

\begin{tabular}{ccccc}
\hline & $\mathbf{E}_{\mathbf{1}}(\mathbf{G P a})$ & $\mathbf{E}_{\mathbf{2}}(\mathbf{G P a})$ & $\mathbf{G}_{\mathbf{1 2}}(\mathbf{G P a})$ & $\mathbf{v}_{\mathbf{1 2}}$ \\
\hline Twintex & 21.5 & 6.0 & 1.00 & 0.20 \\
Plytron & 28.0 & 3.5 & 1.39 & 0.35 \\
CF Epoxy & 104.6 & 6.9 & 3.67 & 0.32 \\
\hline
\end{tabular}

Table 3 - Distribution of chord length and aerodynamic twist angle along the blade length.

\begin{tabular}{ccc}
\hline Location $(\boldsymbol{l} / \boldsymbol{L})$ & Chord $(\boldsymbol{c} / \boldsymbol{L})$ & Twist $\left(^{\circ}\right)$ \\
\hline-0.1 & 0.109 & 23.5 \\
0.0 & 0.107 & 21.9 \\
0.1 & 0.083 & 18.6 \\
0.2 & 0.067 & 15.5 \\
0.3 & 0.056 & 12.6 \\
0.4 & 0.049 & 9.9 \\
0.5 & 0.045 & 7.5 \\
0.6 & 0.044 & 5.3 \\
0.7 & 0.044 & 3.4 \\
0.8 & 0.043 & 1.7 \\
0.9 & 0.042 & 0.2 \\
1.0 & 0.000 & -1.1 \\
\hline
\end{tabular}

Table 4 - Design variables, their limits and values for the bespoke blade design. TE and $L E$ refer to the trailing edge and leading edge laminates, respectively.

\begin{tabular}{ccccc}
\hline Name & Description & Min & Max & Bespoke \\
\hline$x_{1}$ & Number of Spar Cap Plies Section 1 & 1 & 20 & 20 \\
$x_{2}$ & Number of Spar Cap Plies Section 2 & 1 & 20 & 20 \\
$x_{3}$ & Number of Spar Cap Plies Section 3 & 1 & 20 & 20 \\
$x_{4}$ & Number of Spar Cap Plies Section 4 & 1 & 20 & 16 \\
$x_{5}$ & Number of Spar Cap Plies Section 5 & 1 & 20 & 8 \\
$x_{6}$ & Number of Outer Skin Plies Section 1 & 2 & 20 & 2 \\
$x_{7}$ & Number of Outer Skin Plies Section 2 & 2 & 20 & 2 \\
$x_{8}$ & Number of Outer Skin Plies Section 3 & 2 & 20 & 2 \\
$x_{9}$ & Number of Outer Skin Plies Section 4 & 2 & 20 & 2 \\
$x_{10}$ & Number of Outer Skin Plies Section 5 & 2 & 20 & 2 \\
$x_{11}$ & Number of TE Plies Section 1 & 0 & 20 & 0 \\
$x_{12}$ & Number of TE Plies Section 2 & 0 & 20 & 2 \\
$x_{13}$ & Number of TE Plies Section 3 & 0 & 20 & 1 \\
$x_{14}$ & Number of TE Plies Section 4 & 0 & 20 & 1 \\
$x_{15}$ & Number of TE Plies Section 5 & 0 & 20 & 1 \\
$x_{16}$ & Number of LE Plies Section 1 & 0 & 20 & 0 \\
$x_{17}$ & Number of LE Plies Section 2 & 0 & 20 & 2 \\
$x_{18}$ & Number of LE Plies Section 3 & 0 & 20 & 0 \\
$x_{19}$ & Number of LE Plies Section 4 & 0 & 20 & 1
\end{tabular}




\begin{tabular}{ccccc}
$x_{20}$ & Number of LE Plies Section 5 & 0 & 20 & 1 \\
$x_{21}$ & Number of Shear Web Plies Section 1 & 1 & 20 & 19 \\
$x_{22}$ & Number of Shear Web Plies Section 2 & 1 & 20 & 15 \\
$x_{23}$ & Number of Shear Web Plies Section 3 & 1 & 20 & 15 \\
$x_{24}$ & Number of Shear Web Plies Section 4 & 1 & 20 & 15 \\
$x_{25}$ & Number of Shear Web Plies Section 5 & 1 & 20 & 10 \\
$x_{26}$ & Ply Drop Location 1 (mm) & 100 & $x_{27}-100$ & 2310 \\
$x_{27}$ & Ply Drop Location 2 (mm) & $x_{26}+100$ & $x_{28}-100$ & 2500 \\
$x_{28}$ & Ply Drop Location 3 (mm) & $x_{27}+100$ & $x_{29}-100$ & 3035 \\
$x_{29}$ & Ply Drop Location 4 (mm) & $x_{28}+100$ & 3650 & 3150 \\
$x_{30}$ & Spar Cap Width (mm) & 60 & 150 & 145 \\
\hline
\end{tabular}

Table 5 - The mass and tip deflection values for the original two test blades (GFPP and CFPP) [21] and the bespoke blade determined by the genetic algorithm.

\begin{tabular}{ccc}
\hline Blade & $\boldsymbol{M}(\boldsymbol{x})(\mathbf{k g})$ & $\boldsymbol{\delta}_{\boldsymbol{t i p}}(\boldsymbol{x}) / \boldsymbol{L}$ \\
\hline Original CFPP (Experimental) & 21.5 & 0.055 \\
Original GFPP (Experimental) & 18.5 & 0.188 \\
Bespoke GFPP (FE) & 20.4 & 0.131 \\
\hline
\end{tabular}

\section{Figures}

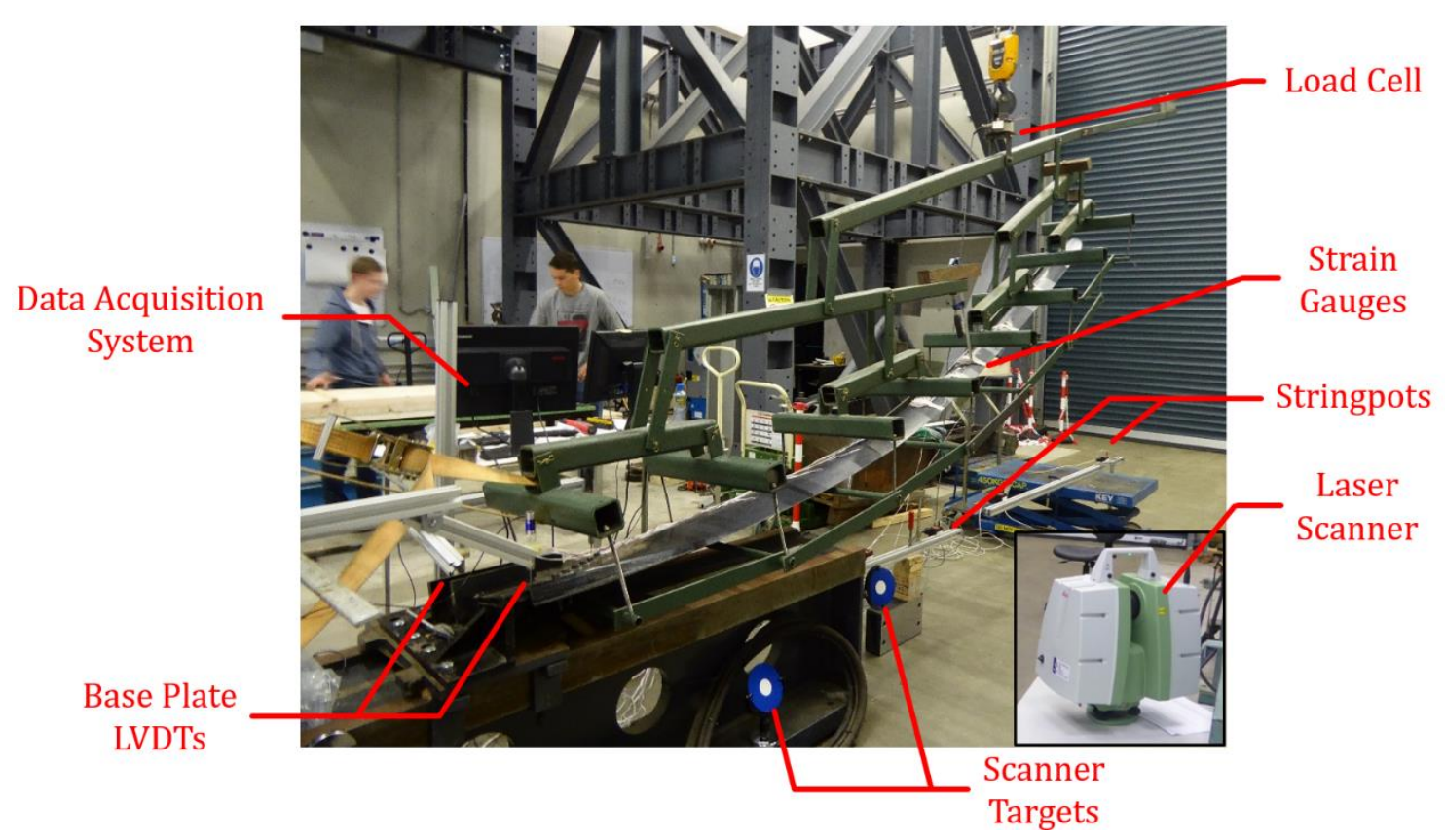

Figure 1 - The Whiffle tree rig applied the load to the blade at eight locations distributed along its length. The instrumentation used in the test included: strain gauges, draw-wire displacement sensors, LVDTs, a load cell and a laser scanner. 

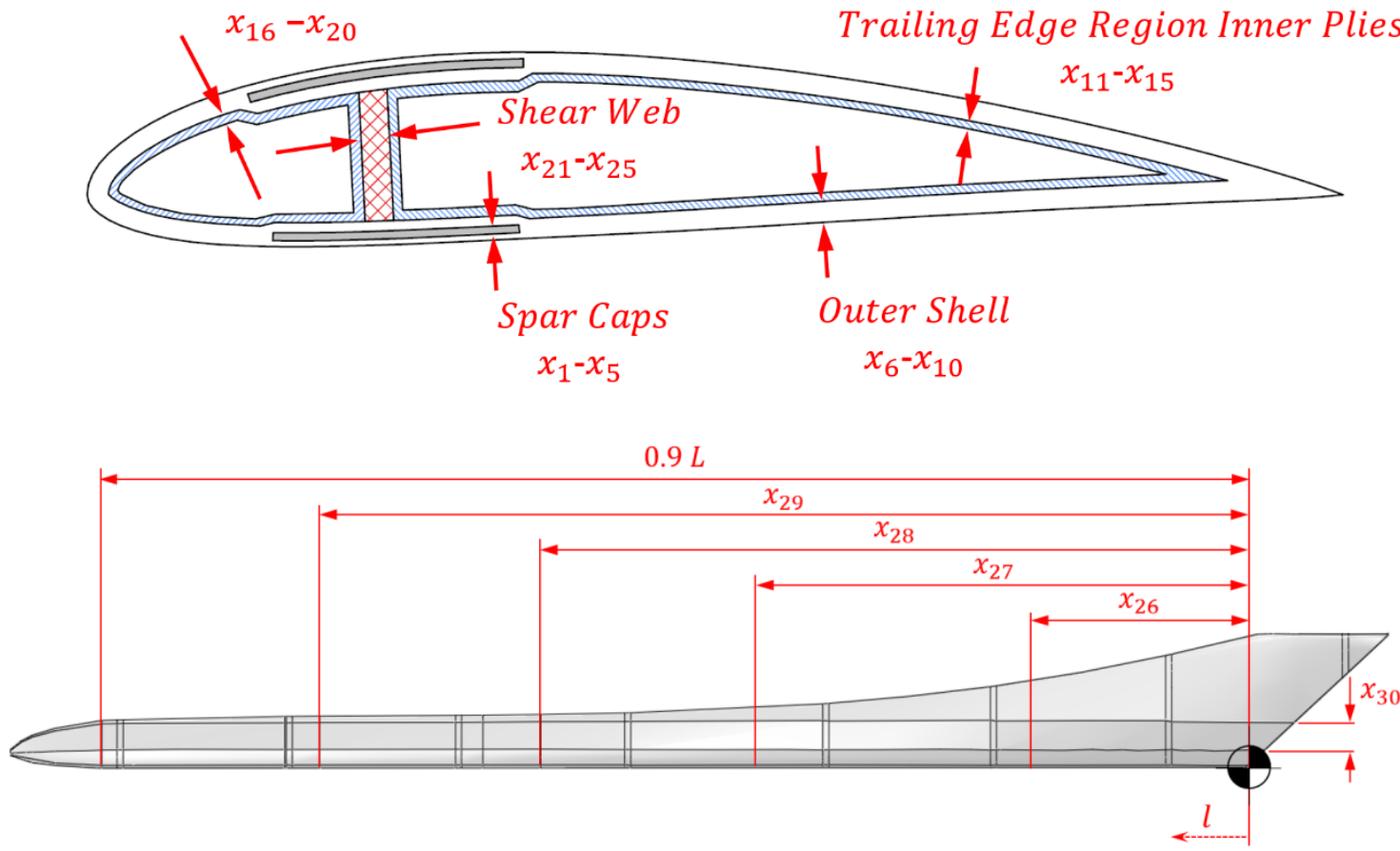

Figure 2 - The design variables control the thickness of the main regions of the blade $\left(x_{1}\right.$ to $\left.x_{25}\right)$, the ply drop locations $\left(x_{26}\right.$ to $\left.x_{29}\right)$ and the width of the spar caps $\left(x_{30}\right)$ in the optimisation procedure.

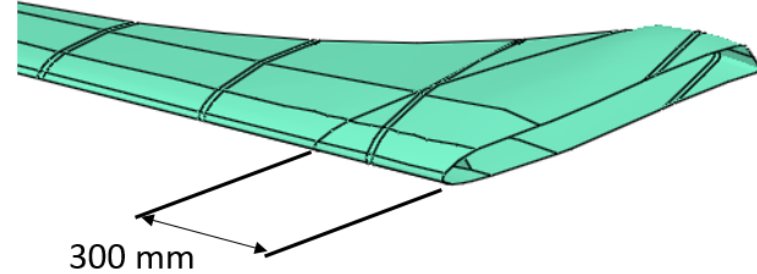

(a) Reinforcement at Root

(b) Reducing Spar Cap Width at Trailing Edge

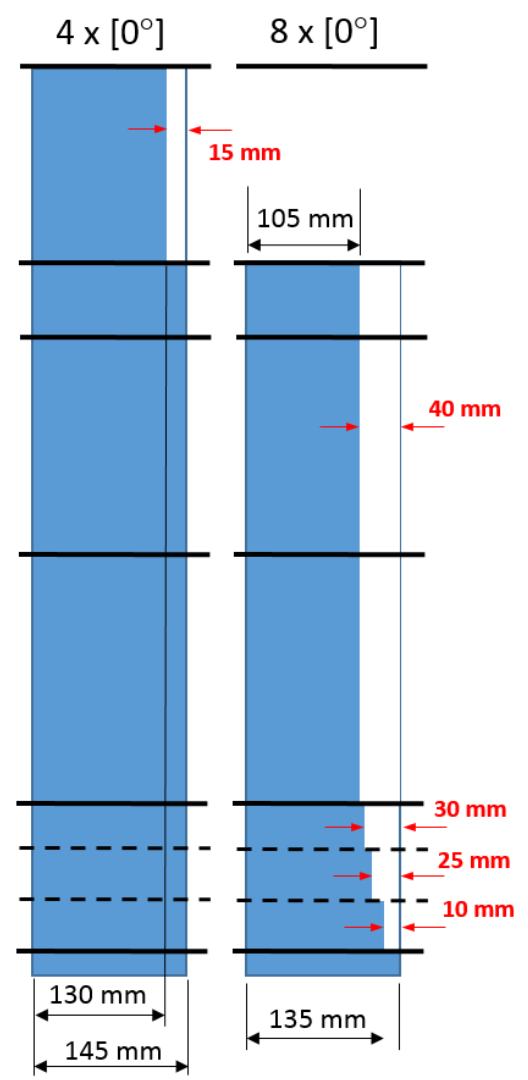

Figure 3 - The blade design was modified to improve manufacturability. (a) The root region of the blade was reinforced with additional biaxial Twintex plies and (b) the width of the spar caps was decreased on the trailing edge side. 


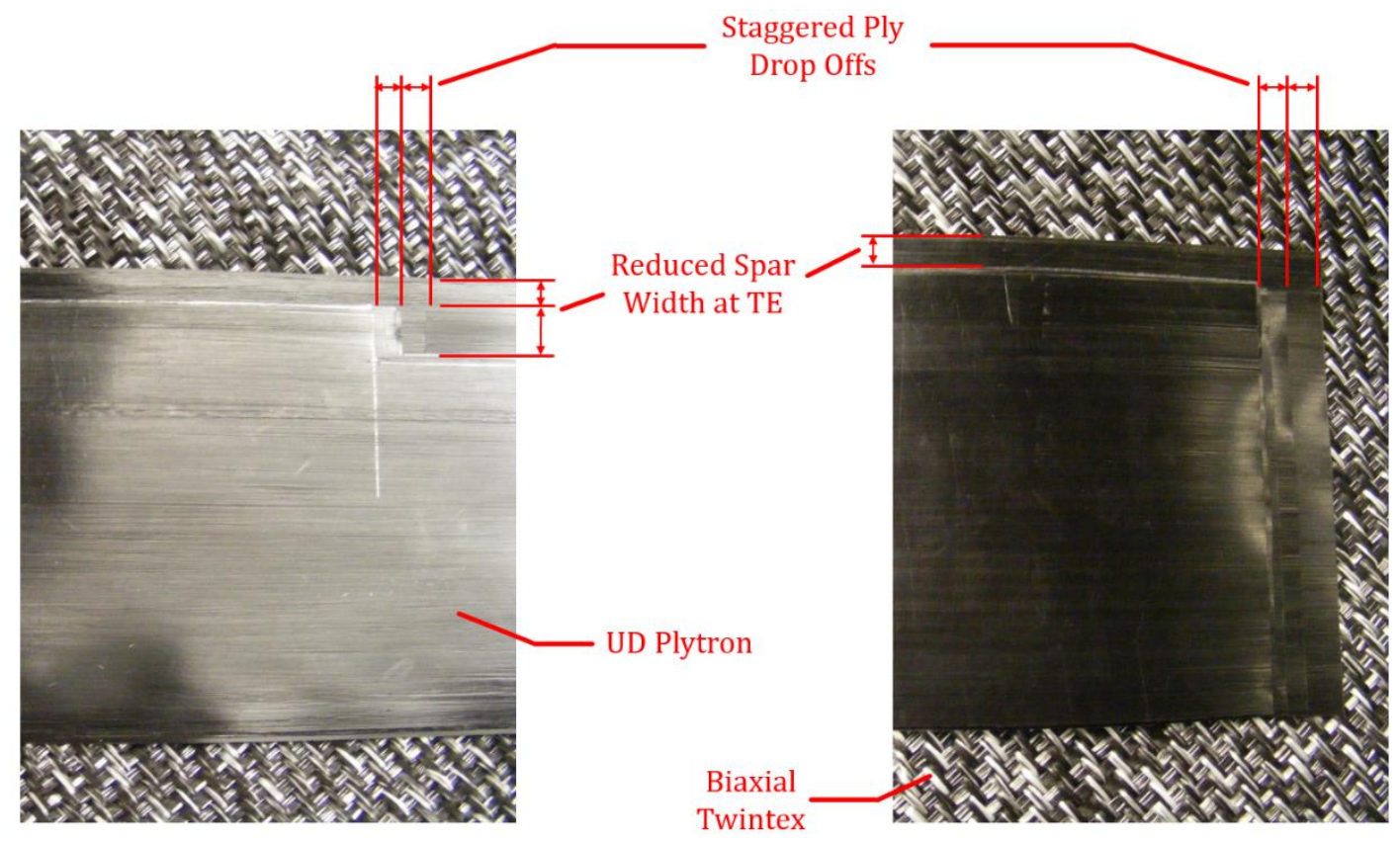

Figure 4 - Photos of the modifications to the spar cap plies, including: staggered ply drops to avoid stress concentrations and reduced width of the spar caps on the trailing edge side of the blade.

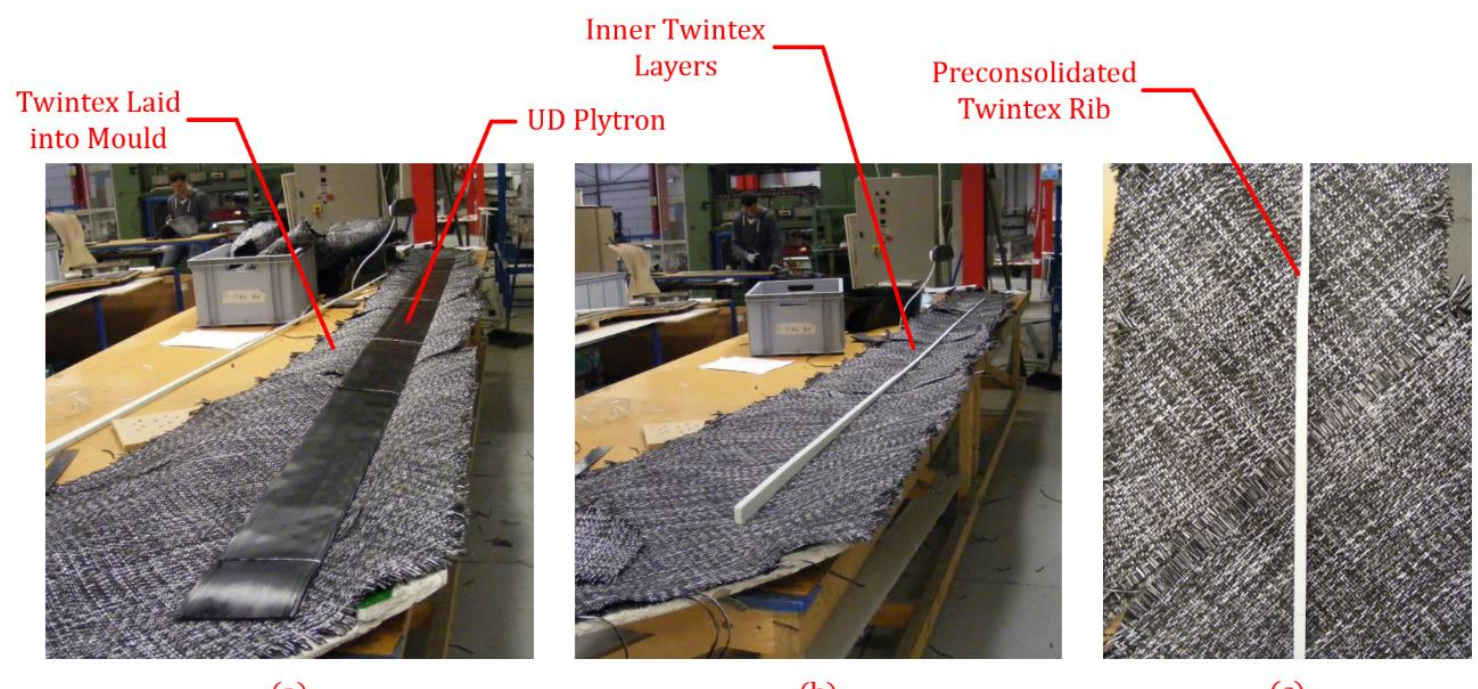

(a)

(b)

(c)

Figure 5 - The initial steps in the blade layup procedure. The outer layers of Twintex were laid into the mold followed by the UD Plytron (a), the internal layers of Twintex were then laid up (b), followed by the preconsolidated shear web (c). 


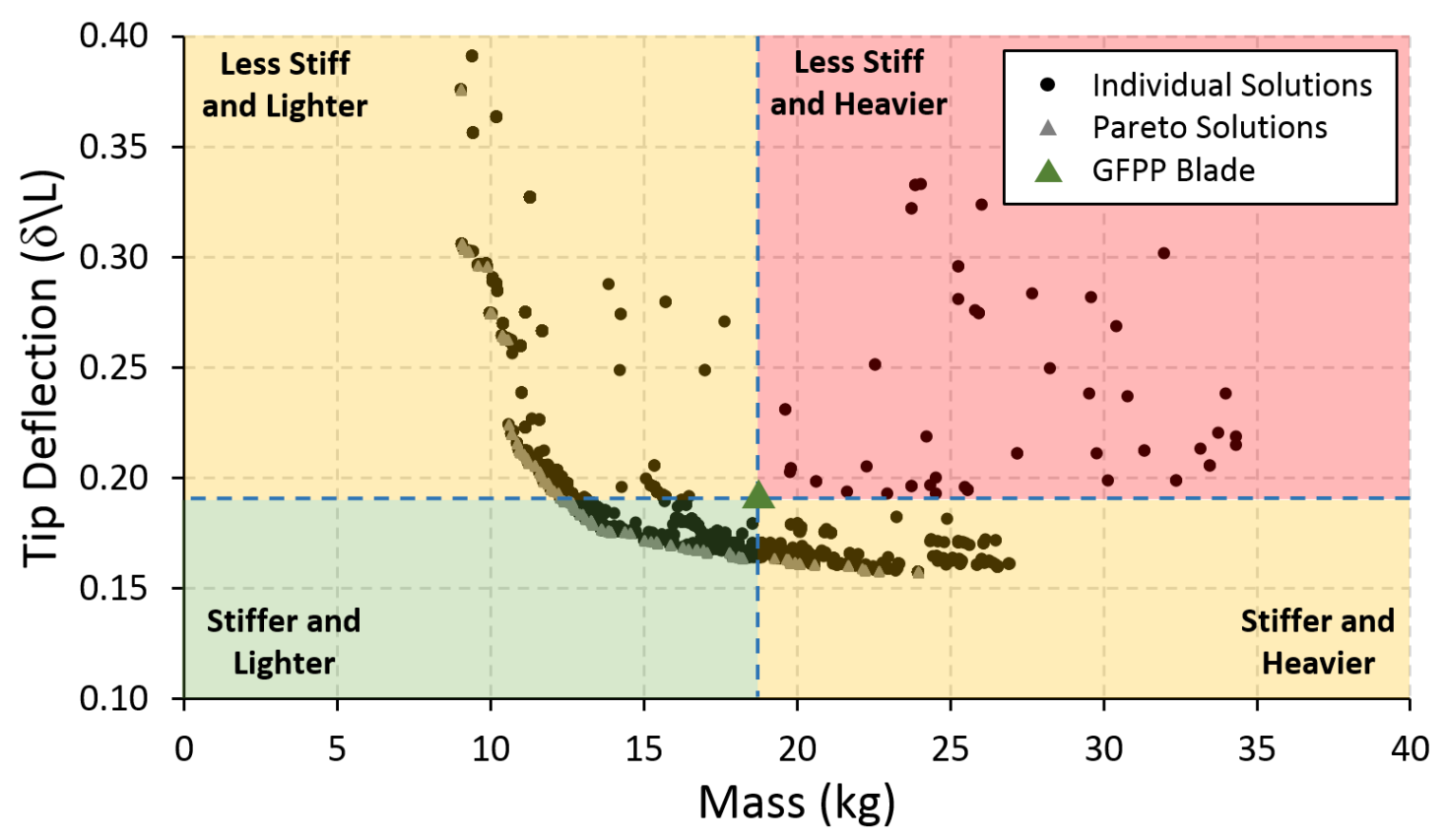

Figure 6 - Results from the previous multi-objective optimisation study [17]. The GFPP blade is noted by the green triangle. Lines of constant mass and constant stiffness split the solution space into quadrants that aid in identifying a new blade design.

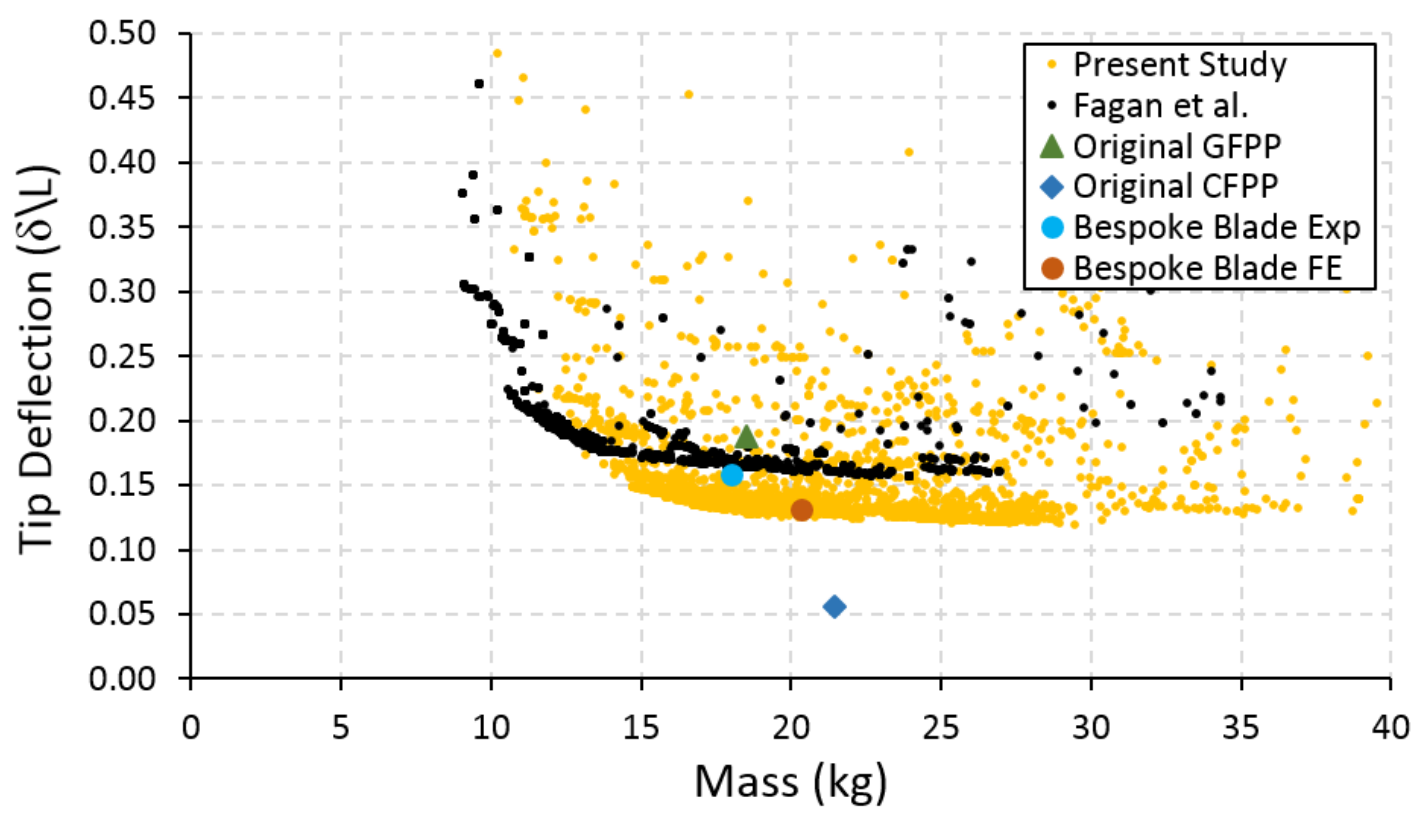

Figure 7 - Results from the multi-objective optimisation of the present (yellow markers) and previous (black markers) studies. The test results of the GFPP and CFPP blades are denoted by the green triangle and blue diamond, respectively. The orange circle indicates the blade design chosen for manufacture, resulting from the present study and the blue circle represents the experimental results from the manufactured blade. 


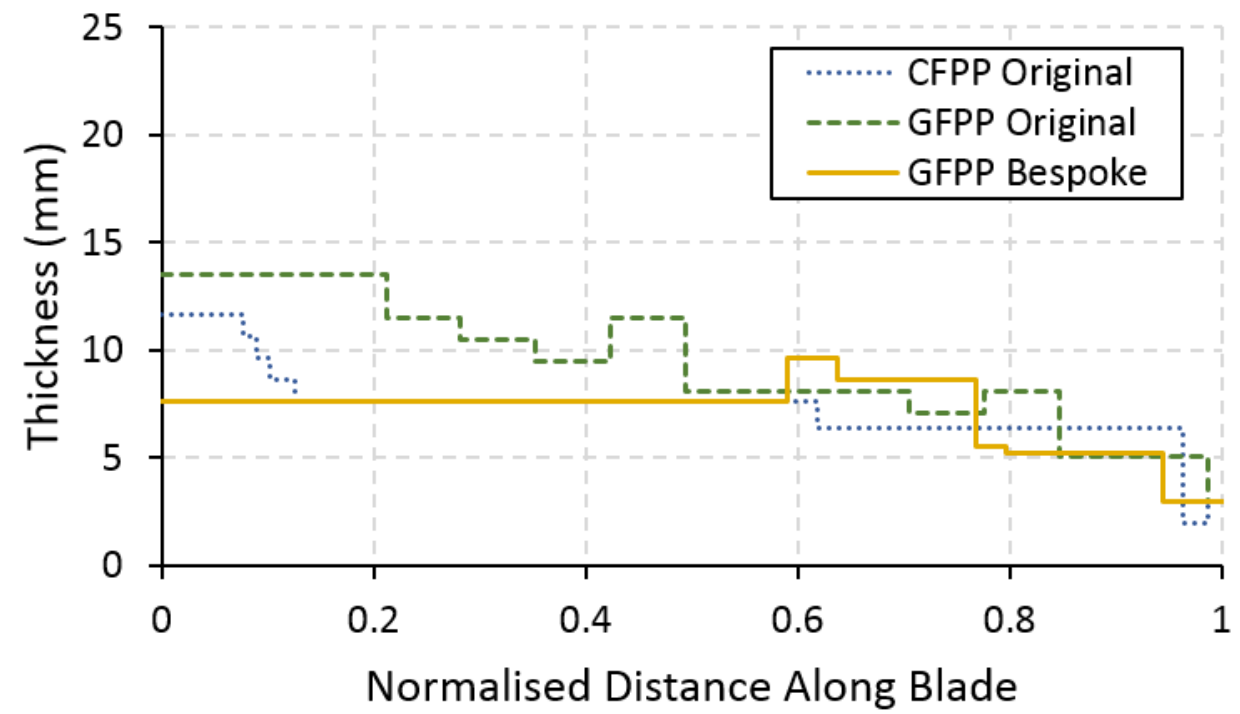

Figure 8 - Comparison of the thickness of the laminates along the centreline of the three blades from the FE models.

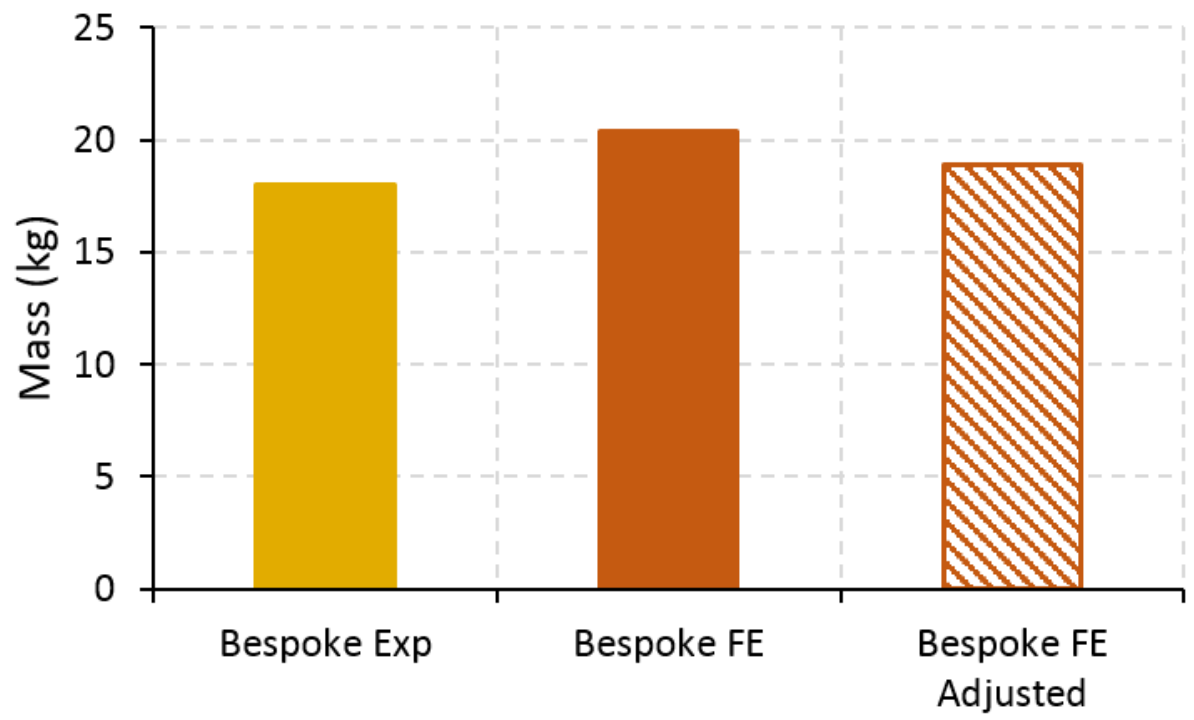

Figure 9 - The experimental results and FE prediction of the blade mass. The value of the FE model prediction adjusted to reflect the manufacturing modifications is also included. 


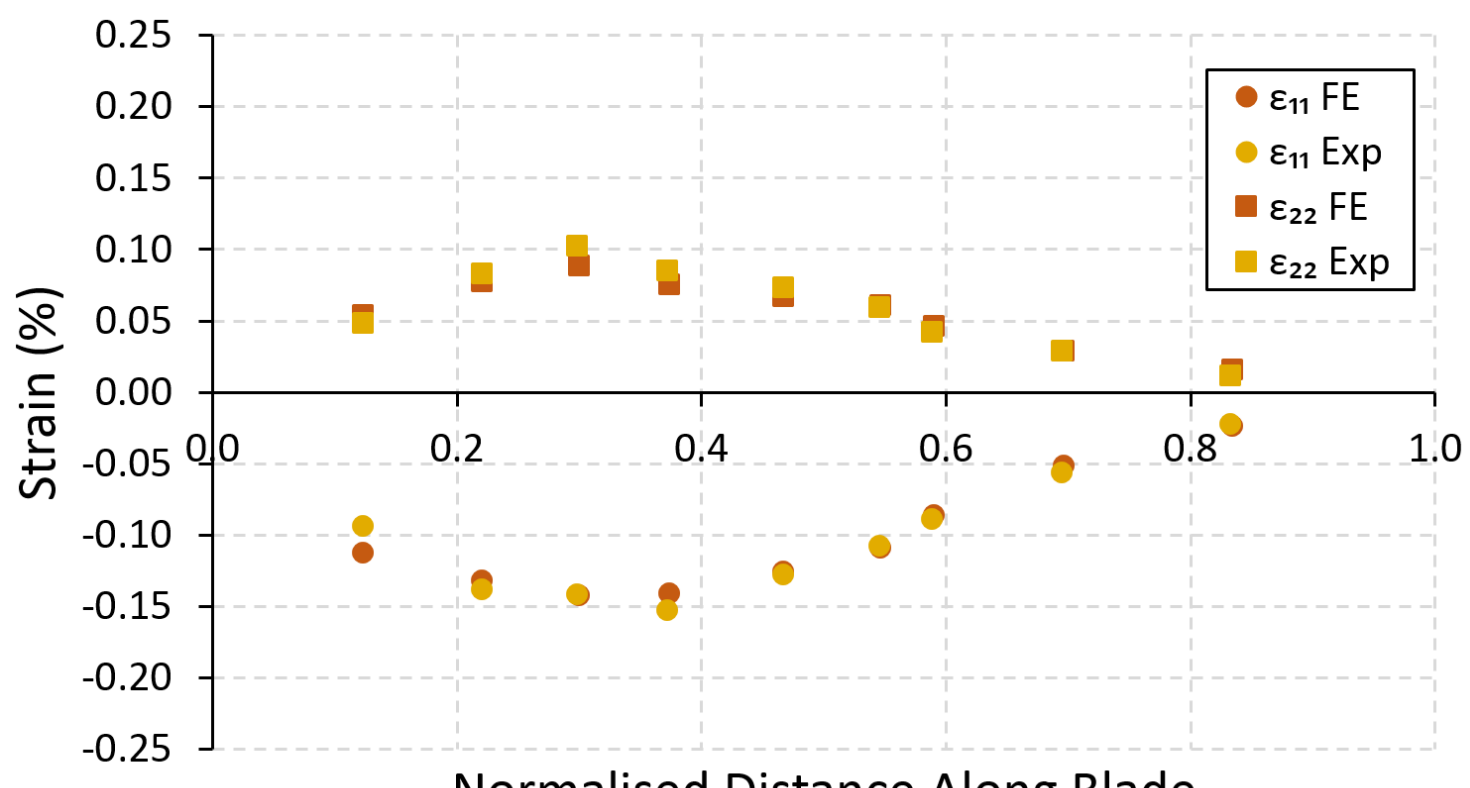

\section{Normalised Distance Along Blade}

Figure 10 - The experimental results and FE prediction of the longitudinal and transverse strain at 9 locations along the blade.

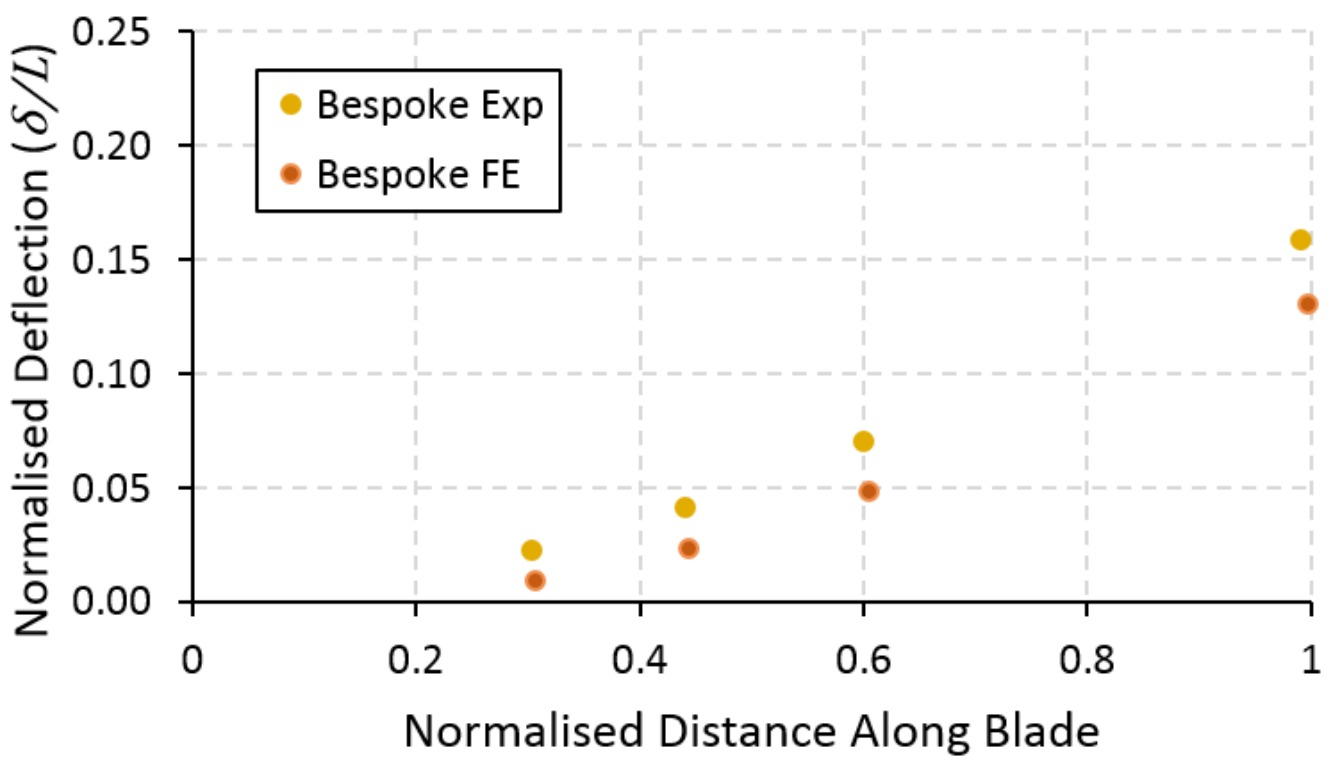

Figure 11 - The experimental results and FE prediction of the deflection along the length of the blade. 


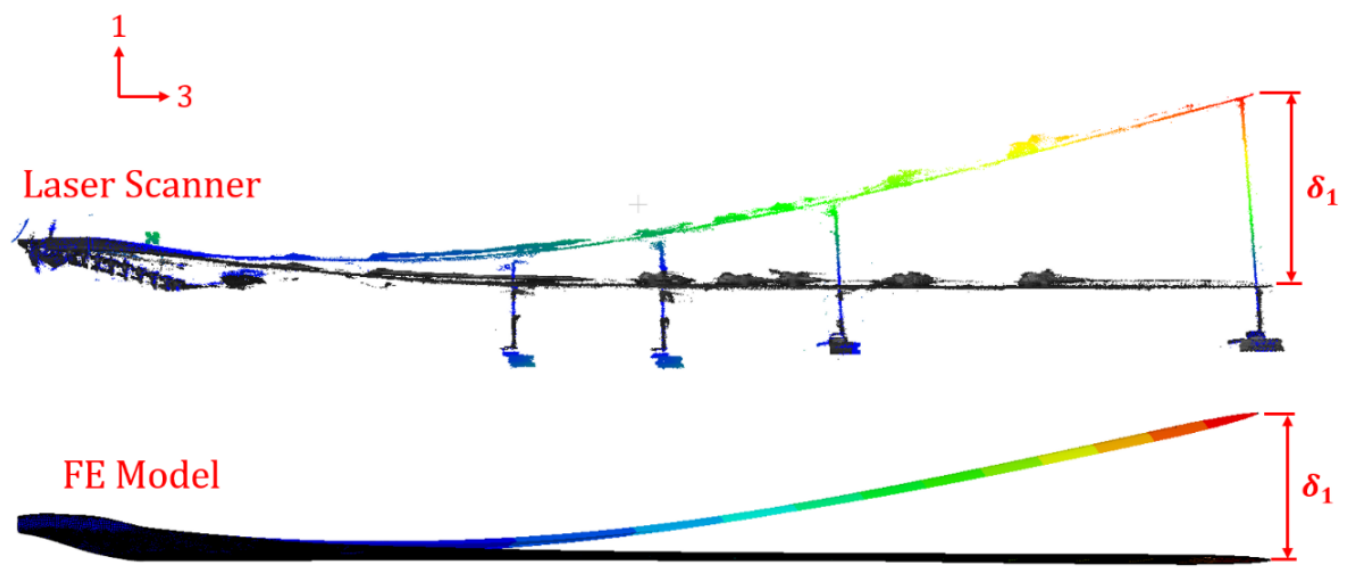

(a)
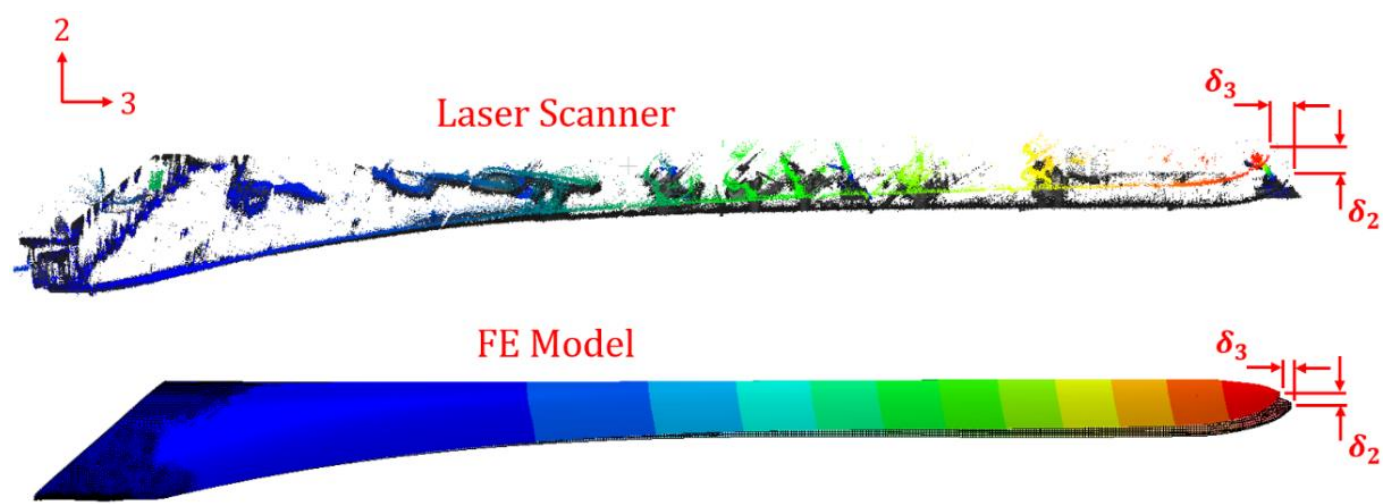

(b)
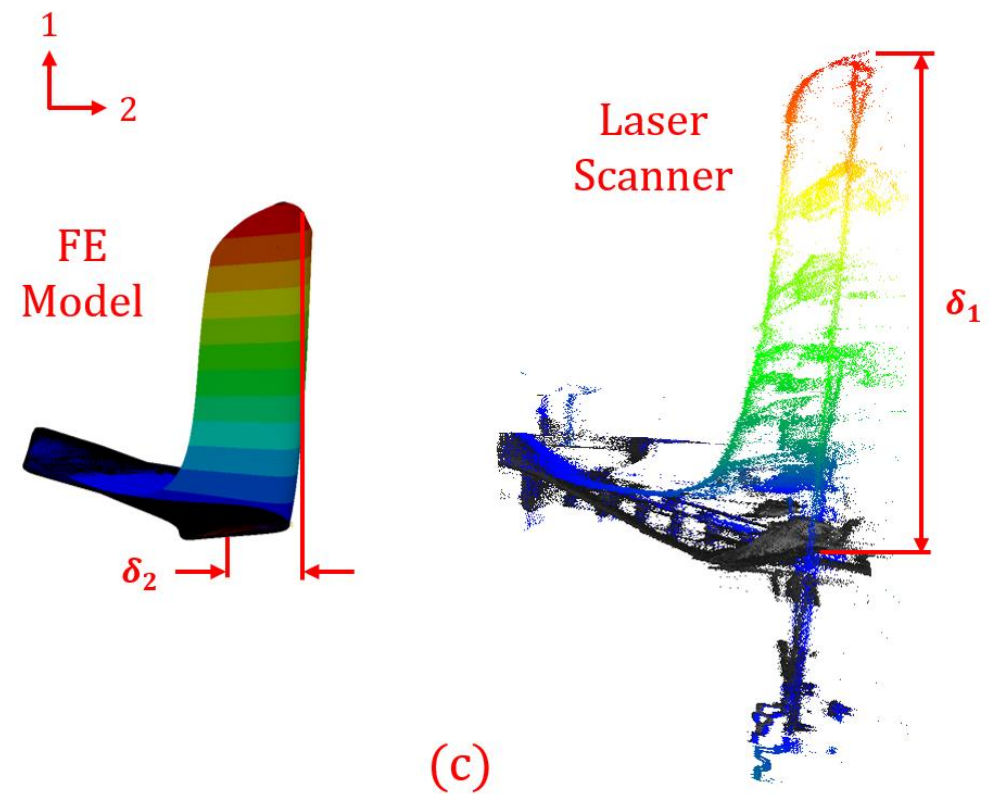

Figure 12 - Point cloud results from the laser scans during testing and FE model deflection results. The unloaded and loaded blades are overlaid and the deflection of the tip in three directions is highlighted for $(a)$ the elevation, $(b)$ the plan and $(c)$ the end elevation view of the blade. 


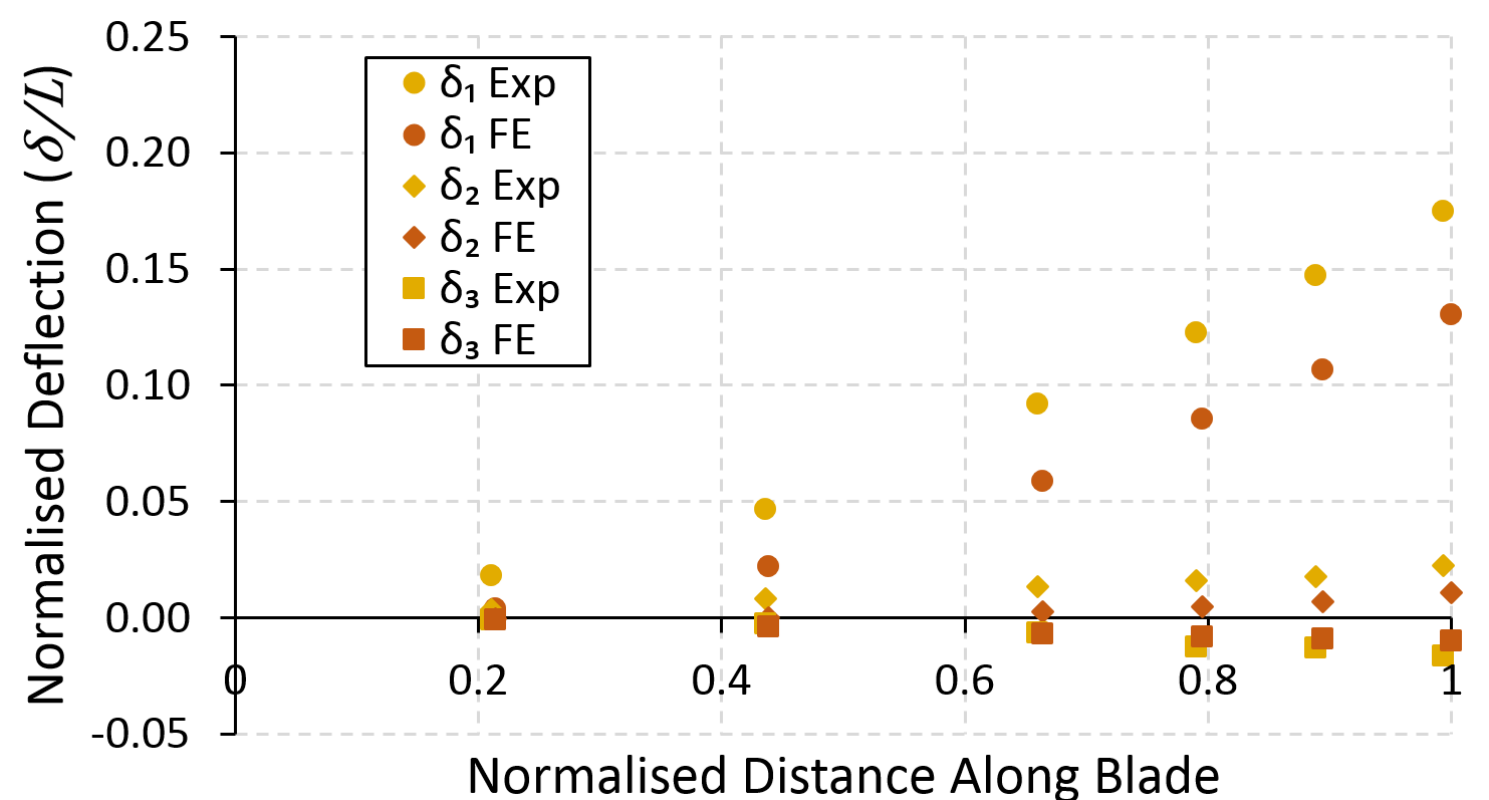

Figure 13 - Comparison between the laser scanner and FE model deflections for six points along the trailing edge of the blade. The indices 1, 2 and 3 refer to the coordinate systems shown in Figure 12.

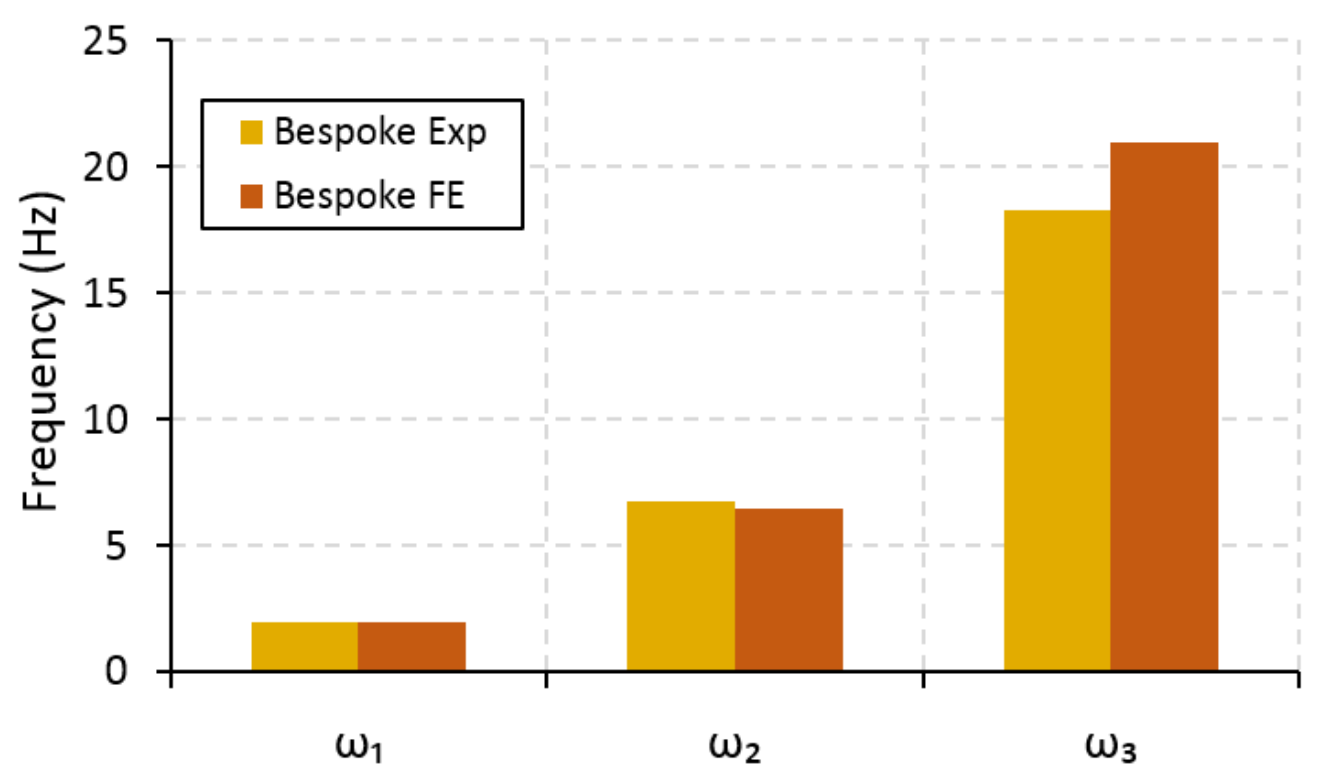

Figure 14 - The experimental results and FE prediction of the first three flapwise bending mode natural frequencies. 


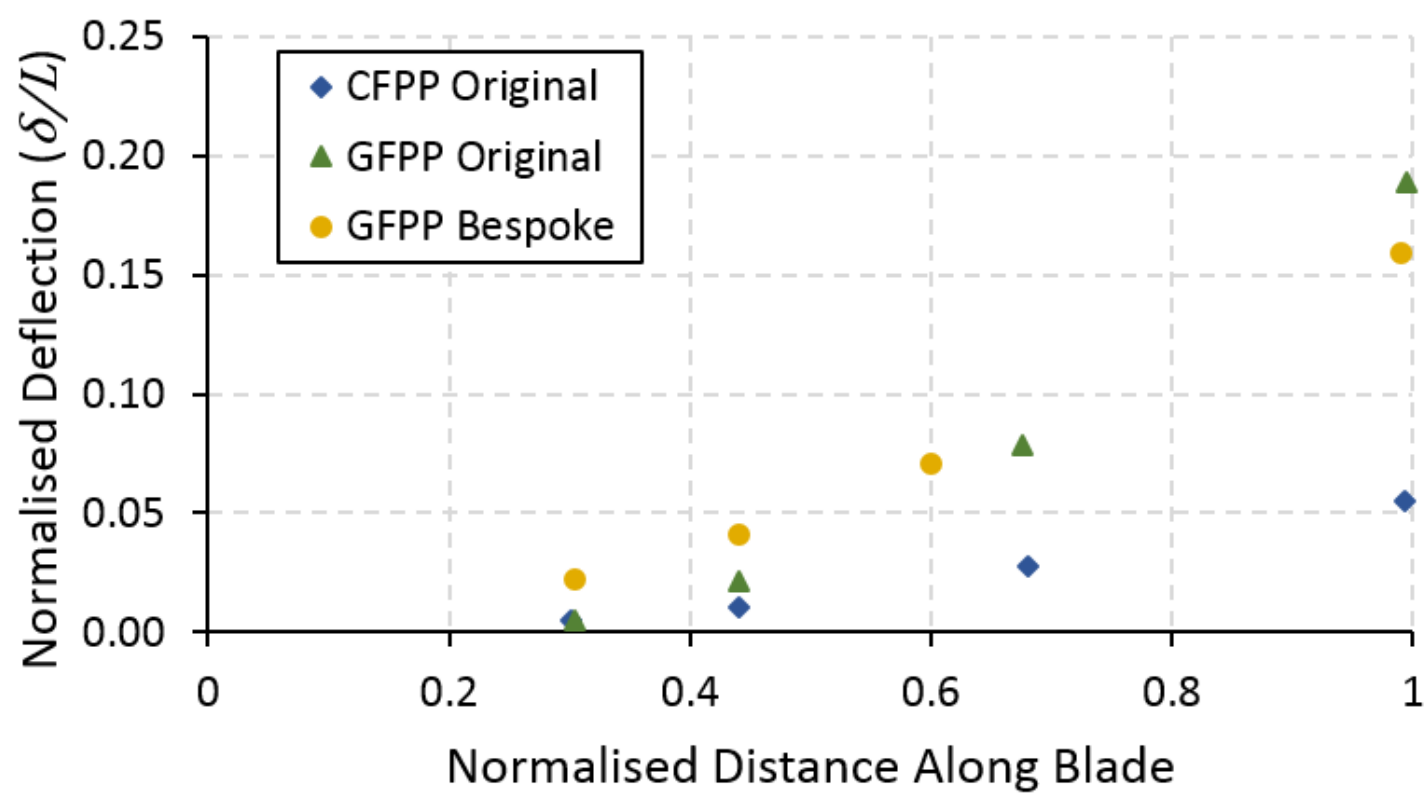

Figure 15 - Blade deflection results from the experiments for the original CFPP, GFPP and new GFPP blades.

Normalised Distance Along Blade

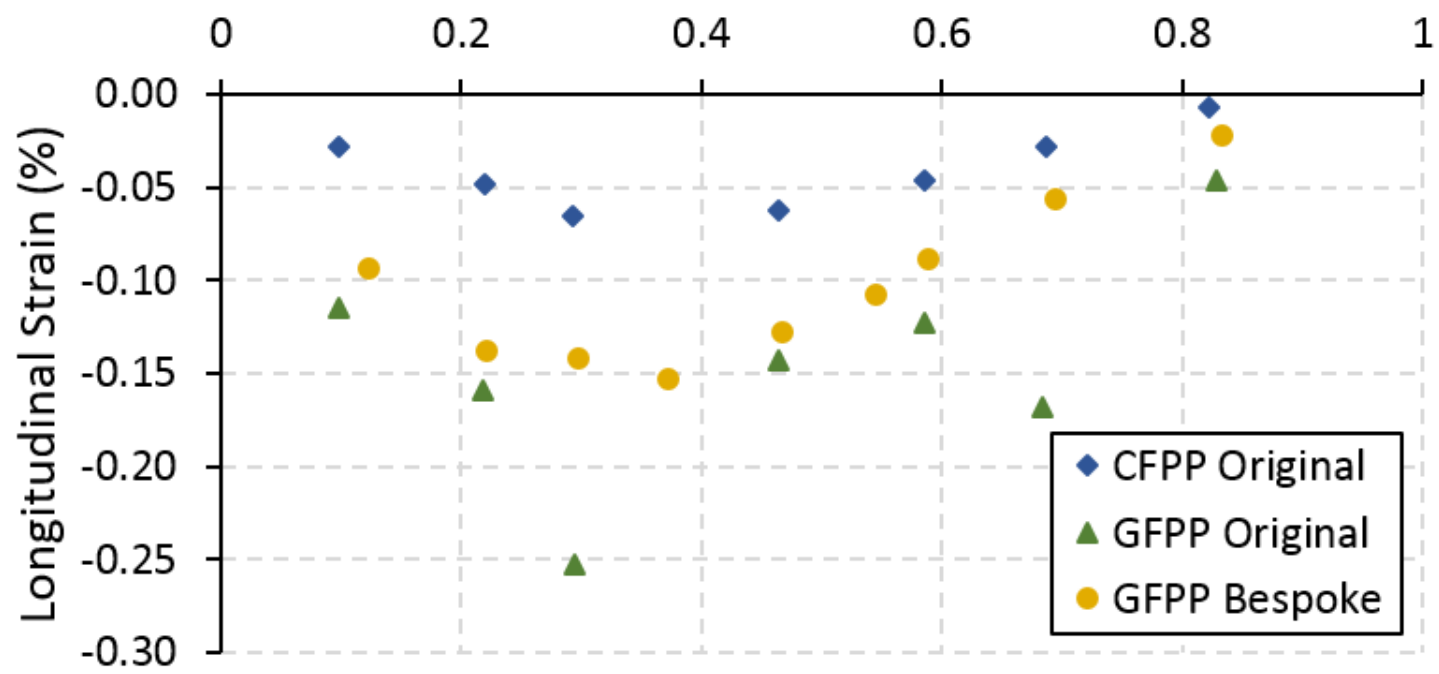

Figure 16 - The longitudinal strain results from the experiments for the original CFPP, GFPP and new GFPP blades. 


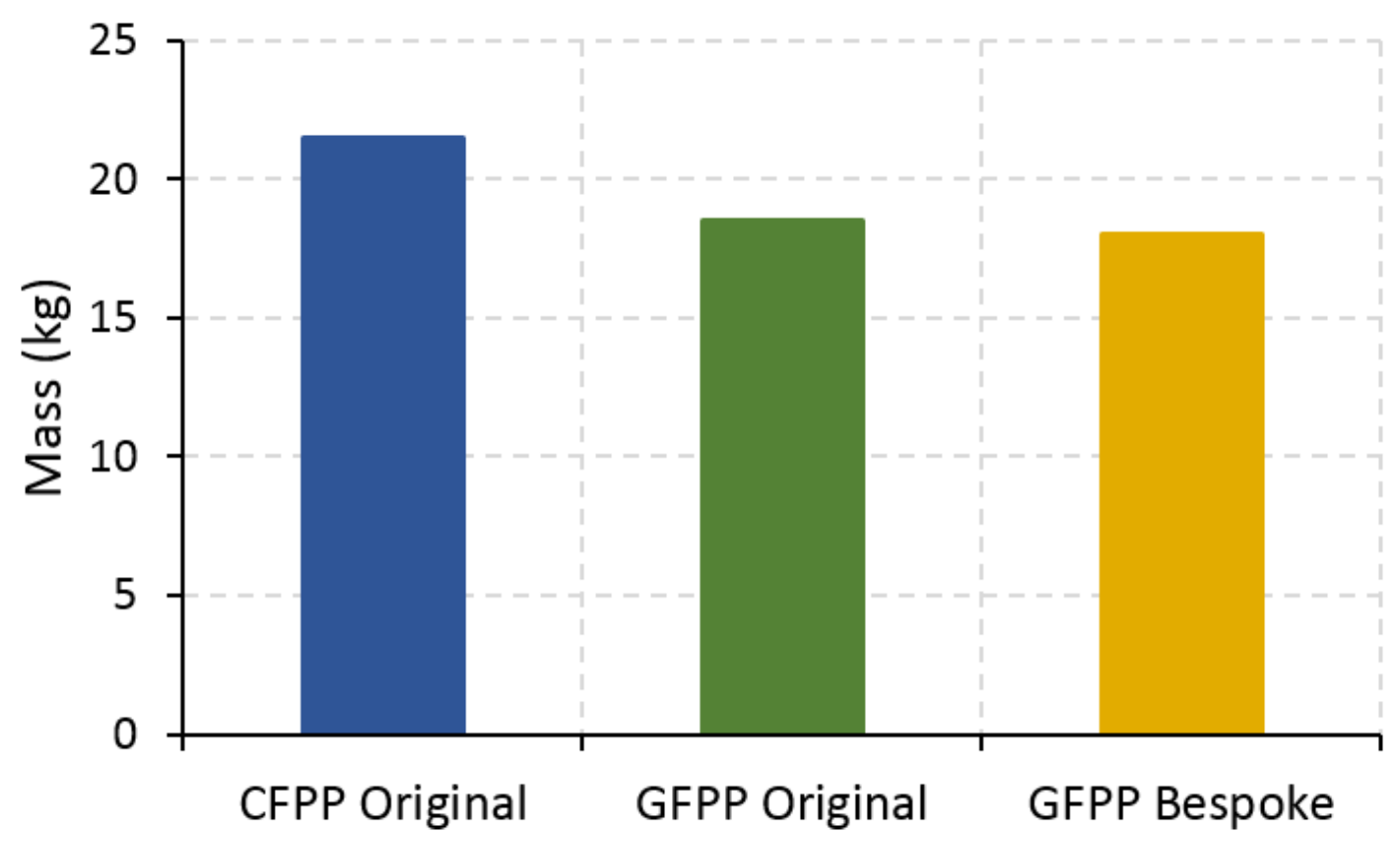

Figure 17 - Blade mass results from the experiments for the original CFPP, GFPP and new GFPP blades.

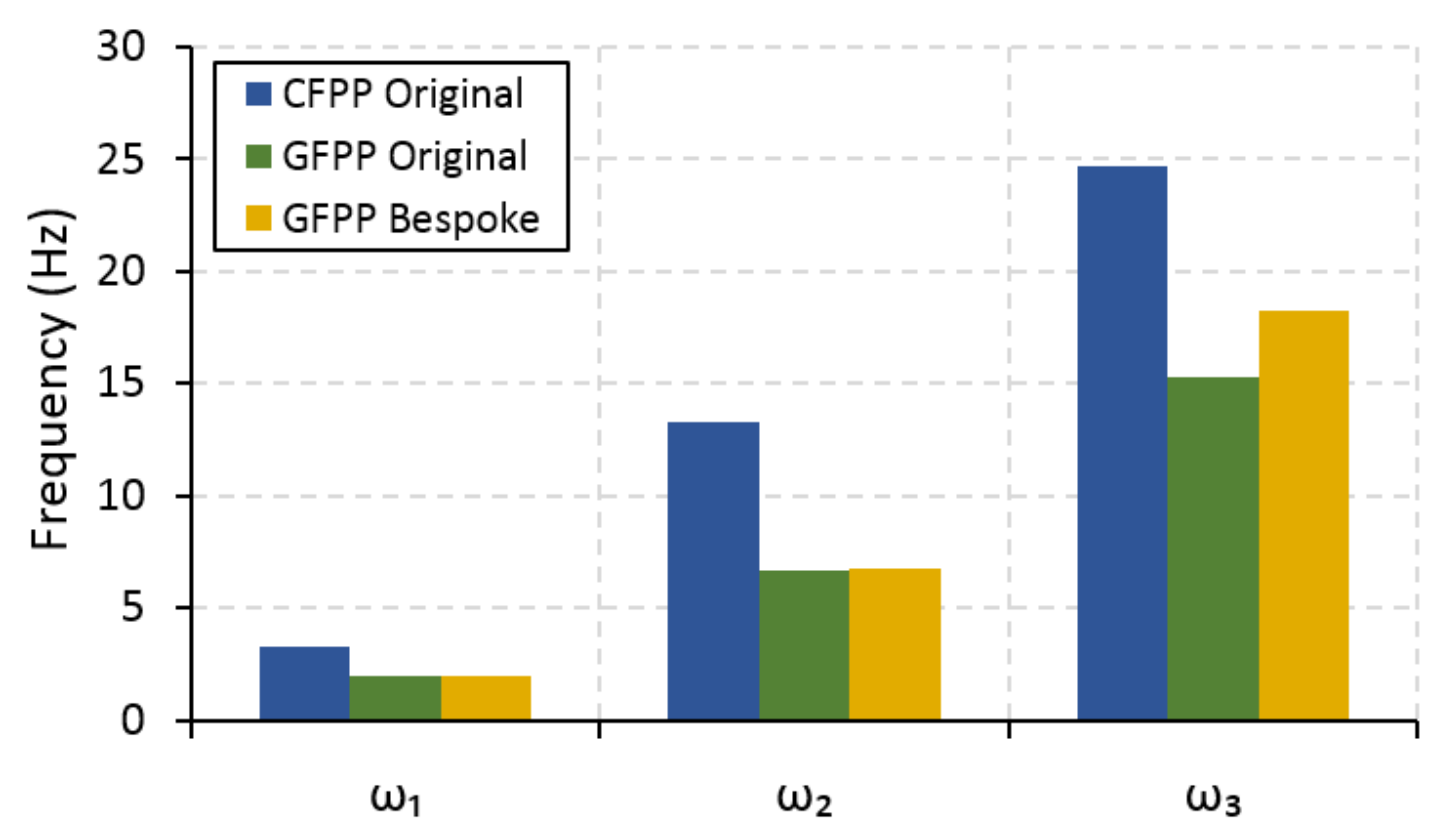

Figure 18 - The first three bending mode natural frequencies from the experiments for the original CFPP, GFPP and new GFPP blades. 